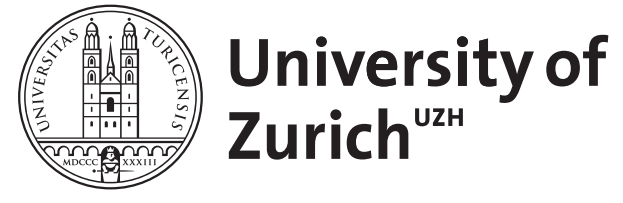

\title{
Zur Funktionsgeschichte
}

Weddigen, Tristan

Posted at the Zurich Open Repository and Archive, University of Zurich

ZORA URL: https://doi.org/10.5167/uzh-74536

Book Section

Published Version

Originally published at:

Weddigen, Tristan (2003). Zur Funktionsgeschichte. In: Weddigen, Tristan; De Blaauw, Sible; Kempers, Bram. Functions and Decorations. Art and Ritual at the Vatican Palace in the Middle Ages and the Renaissance. Vatican City/Turnhout: Brepols, 9-25. 


\section{Tristan Weddigen}

\section{Zur Funktionsgeschichte}

\begin{abstract}
«Der Wegweiser ist in Ordnung, - wenn er, unter normalen Verhältnissen, seinen Zweck erfüllt.» Ludwig Wittgenstein, Philosophische Untersuchungen. ${ }^{1}$
\end{abstract}

In der Kunstgeschichte wird häufig die Frage nach den spezifischen historischen Funktionen von Kunst und Kunstwerken gestellt, ohne dass die Fragestellung selbst hinterfragt würde. Der Zweck der vorliegenden Untersuchung ist es, die Funktionsgeschichte als methodischen Ansatz auszukundschaften. Diese Suche nach einem kunstwissenschaftlichen Funktionsbegriff bietet einen Ausblick auf verschiedene Problemfelder der Praxis und Theorie des Faches und verweist auf Lichtungen und Holzwege, die noch der Erkundung harren. Die Untersuchung, die mit einer Erörterung des Funktionsbegriffs ansetzt, versucht, dessen Stärken und Schwächen, Möglichkeiten und Grenzen in Bezug auf die Kunstwissenschaft auszuloten. Der skizzenhaft kartierte Ansatz der Funktionsgeschichte muss schließlich begriffshistorisch kontextualisiert und konkretisiert werden, um ihn auf die italienische Kunst der frühen Neuzeit anwenden zu können.

\section{<unktion}

Was ist eine «Funktion`? Die vornehmliche Funktion etwa eines Buches ist gelesen zu werden. Es besitzt daher eine Form, welche die Lektüre ermöglicht und dazu anregt. Es ist ein Ding ‘zum〉 Lesen - die Präposition ‘zu〉 weist auf ein Ziel hin, wovon der Gegenstand abhängt und durch das er definiert wird. ${ }^{2}$ Als «Funktion` bezeichnet man die intendierte Handlung und das Ziel, die ein Subjekt mittels eines zweckmäßig geformten Ge-

\footnotetext{
${ }^{1}$ Wittgenstein 1984, 290, no. 87

Beim vorliegenden Beitrag handelt es sich um ein überarbeitetes Kapitel aus meiner Dissertation, die ich 2001 unter dem Titel Raffaels Papageizimmer - Raumfunktion und Dekoration im Vatikanpalast der Renaissance an der Technischen Universität Berlin eingereicht habe. Der Tagungsvortrag mit dem Titel Sala papagalli vaticana betraf hingegen die funktionsgeschichtlichen Aspekte des vatikanischen Papageizimmers. Ich danke meinen Freunden Sible de Blaauw und Bram Kempers ganz herzlich für ihre jahrelange intellektuelle, moralische und kulinarische Unterstützung, die meinen Romaufenthalt so ersprießlich und ergötzlich gemacht hat. Marianne Koos bin ich dankbar für ihre kritischen Lektüren und für ihre methodologischen Anregungen.
}

genstandes vollführt oder erreicht - oder in den Worten der Encyclopédie (1751-1765): Eine Funktion ist «die Handlung des Mittels, welches dasjenige tut, wozu es bestimmt oder gezwungen ist». ${ }^{3}$

Wie die lateinischen Wörter 〈fungere〉 und 〈functio〉 andeuten, fügt sich einerseits der passive Gegenstand seiner Funktionsbestimmung und verrichtet andererseits aktiv jene Aufgabe, er motiviert und gestaltet teilweise jene Handlung durch seine suggestive Einwirkung auf den Benutzer. Von der Form des gebrauchten Gegenstandes hängen Funktionalität, Angemessenheit, Zweckmäßigkeit ab. Indem Zweckrationalität Unterschiedliches auf ein Handlungsziel hin wertend vergleicht und so in eine Interrelation setzt, humanisiert sie einerseits den Gegenstand und verdinglicht andererseits den Menschen. Eine der Schwierigkeiten beim Sprechen über Funktionen oder Aufgaben von Kunstwerken ist jene anthropozentrische Perspektive, die das Werk, ein bildliches und zeichenhaftes Artefakt, sowohl als dienliches Zeug wie auch als autonomes Lebewesen betrachtet. $^{4}$

Eine intendierte oder faktische Funktion stellt nicht nur eine Relation zwischen Subjekt und Objekt her, sondern Zweckbestimmung und Nutzung sind zugleich integraler Teil der Bedeutung des Gegenstandes, seines Sinnes, nämlich seines Ausgerichtetseins von Etwas auf Etwas anderes. ${ }^{5}$ Es geht weniger um das verortende Warum oder das beschreibende Wieso, sondern mehr um das kausale Weswegen oder das zweckgerichtete Wozu. ${ }^{6}$ In Anlehnung an Ludwig Wittgen-

${ }^{2}$ Cf. HiRdina 2000-2001, 589: «Gegenüber dem Autonomen verweisen Termini im Umfeld von 〈Funktion〉 - unabhängig davon, ob sie in ästhetischem oder nichtästhetischem Kontext stehen - immer auf eine Abhängigkeit: von einem Ziel in der Spanne zwischen pragmatisch und teleologisch; von einer Aufgabe zwischen technologisch und sozial; von einem Problem, das zu lösen ist; von einer Struktur, die Träger von Funktionen ist; von einer Konstanten, die den Spielraum der Variablen bestimmt; von Bedingungen, die gegeben sein müssen, um das Potentielle einer Funktion in einer Aktion, einer Tätigkeit, einem Prozess realisieren zu können.» Ib.: «Funktion» meint allgemein Beziehungen zwischen einem Resultat und seinen Voraussetzungen.» Cf. MitTelstrass et al. 1980-1996, vol. 1, 691: Funktion.

${ }^{3}$ Diderot / d'Alembert 2000, Supplément Panckoucke, Fonction: «Fonction, s. f. (gramm.) c'est l'action de l'agent qui fait la chose à laquelle il est destiné ou obligé.»

${ }^{4}$ Cf. Heidegger 1986, 11-34, cap. Das Ding und das Werk.

${ }^{5}$ Cf. ERLER ET AL. 1971-1995 (Relation) und KöNIg 1971-1995 (Relativismus) 
steins (1889-1951) Beschreibung und Deutung des Wortes «Bedeutung als dessen «Gebrauch in der Sprache», darf eine Zweckrelation als bedeutungsstiftend verstanden werden. ${ }^{7}$ Eine Funktionsanalyse kann die anthropologische Bedeutung des Gegenstandes «Buch» auf Grund der Beschreibung seiner Lektüre definieren, nämlich als einen zu beobachtenden, mehr oder minder normierten Handlungsablauf, der zu anderen Zuständen überleitet. ${ }^{8}$

So wie «Bedeutung ist auch «Funktion〉 ein Begriff kommunikativer oder gesellschaftlicher Art. ${ }^{9}$ Objekte entstehen in einem sozialen oder kulturellen Beziehungsgewebe und erhalten darin ihre jeweilige Form, Funktion und Bedeutung. ${ }^{10}$ Als Gegenstände erfüllen auch Bilder und Kunstwerke Zwecke, nach welchen denen gemäß sie gestaltet sind, und ermöglichen oder veranlassen dank ihrer Form konkrete Gebrauchsweisen. Bilder, die etwa der religiösen Andacht dienen, unterscheiden sich in ihrer Form und Bedeutung zweckgemäß von solchen, die der wissenschaftlichen Illustration nützen sollen. ${ }^{11}$

\section{〈Funktionsgeschichte〉}

Objekte sind nicht nur gesellschaftlich eingebunden und polyfunktional, sondern ihre Funktionen verändern sich auch in der Zeit. Die geschichtliche Bedeutung

${ }^{6}$ Cf. Hirdina 1990, 214: «Funktionalismus bezeichnet in den Wissenschaften eine theoretische Methode (funktionale Methode), bei der jeweilige Phänomene in ihrer kausalen Abhängigkeit erklärt und vor allem in Bezug auf ihre Wirkung untersucht werden.»Cf. FRICKE 1997-2000, 643: zum Funktionsbegriff in der Literaturwissenschaft - «ein Text beziehungsweise ein Textelement erfüllt eine bestimmte Funktion [...], wenn es die in empirischer Verallgemeinerung nachweisbare Disposition [...] besitzt, angebbare Textrelationen herzustellen und Lesewirkungen hervorzurufen. Dafür ist es unerheblich, ob dies auf einer unterstellten Wirkungsabsicht des Autors beruht und ob in jedem Einzelfall die entsprechende Wirkung auch tatsächlich eintritt.»

${ }^{7}$ Cf. Wittgenstein 1984, 262, no. 43: «Man kann für eine große Klasse von Fällen der Benützung des Wortes 〈Bedeutung〉 - wenn auch nicht für alle Fälle seiner Benützung - dieses Wort so erklären: Die Bedeutung eines Wortes ist sein Gebrauch in der Sprache.» Cf. ib., 214 , no. 10

${ }^{8}$ Cf. RAdCLIFFE-Brown 1968 und Louis Marin ib., 34-38: zum anthropologischen Funktionsbegriff.

${ }^{9}$ Cf. Luhmann 1998, 224: «Wie alle in der Gesellschaft anfallenden Funktionen (ob zu Systemen ausdifferenziert oder nicht) geht auch die Funktion der Kunst letztlich auf Probleme sinnhafter Kommunikation zurück. Sie dient als Medium der Kommunikation, aber auch als Medium des Bewusstseins.» Cf. GEERTz 1987b, 18 : «Kultur ist deshalb öffentlich, weil Bedeutung etwas Öffentliches ist.»

${ }^{10}$ Cf. Geertz 1987в, 9: «Ich meine mit Max Weber, dass der Mensch ein Wesen ist, das in selbstgesponnene Bedeutungsgewebe verstrickt ist, wobei ich Kultur als dieses Gewebe ansehe.»

${ }^{11}$ Cf. z. B. Belting 1995, 100: zur Funktionalität des Andachtsbildes. eines Gegenstandes kann daher in seinen historischen Funktionen erkannt und anhand ihrer dargestellt werden. Eine solche Betrachtungsweise ließe sich «Funktionsgeschichte> nennen und unter diesem Begriff in der Kunstwissenschaft einführen. ${ }^{12}$ Der nachvollziehbare Zweck und dokumentierte Gebrauch eines Gegenstandes helfen, dessen historisch überlieferte Form zu erklären, so wie beispielsweise liturgische und weltliche Nutzungsweisen die Architekturform gotischer Kathedralen begreiflich machen können. ${ }^{13}$

Die funktionsgeschichtliche Analyse von Bildern und Kunstwerken, die der Kulturhistoriker Jacob Burckhardt (1818-1897) als kunstgeschichtliche Darstellung «nach Aufgaben» - als Ergänzung zur Künstlerbiografik - einführte, hat sich, nach Hans Belting, in der sozialhistorisch orientierten Forschung seit der Mitte des 20. Jahrhunderts unter dem sprachwissenschaftlich geprägten Oberbegriff 〈Kunst im Kontext〉 etabliert. ${ }^{14}$ Während Kunstgeschichte «nach Aufgaben» auf die Rekonstruktion vor allem künstlerischer Intentionen abzielte, umfasst die spätere Kontextforschung auch die künstlerische Rezeption als Beleg für den tatsächlichen Gebrauch von Kunstwerken. ${ }^{15}$ Wie Ján Bakoš gezeigt hat, wurde freilich dieses ambivalente Erbe Burckharts, nämlich die "Aufgaben» einer zugleich auto- wie auch heteronom verstandenen Kunst, sowohl zur Begründung formalistischer Stilgeschichte als auch sozialgeschichtlicher Ansätze in Anspruch genommen. ${ }^{16}$ Die Kontextualisierung des künstlerischen Einzelwerkes in-

${ }^{12}$ Cf. Belting 1986, Baschet 1996, Busch 1997c: zur Funktionsgeschichte der Kunst.

${ }^{13}$ Cf. z. B. Kimpel / Suckale 1997A, id. 1997b und SAuerLäNder 1997: zur funktionalen Genese gotischer Kathedralen.

${ }^{14}$ WöLfFLIN 1947A, 143: «Die Kunst nach Aufgaben, das ist mein Vermächtnis.» WöLFFLIN 1947в, 149: «nach Sachen und Gattungen». Ib. 150-151: «Kunst nach Aufgaben». Cf. Huse 1977: zu Jacob Burckhardt. Belting 1986: zur Kunst im Kontext. Cf. Didi-HuberMAN 1996, 60-62: Der kunsthistorische Funktionsbegriff sei sozialhistorisch geprägt und durch Ernst Cassirers Begriff «symbolische Funktion> in den Kreis Aby M. Warburgs eingeführt worden. Cf. GOMBRich 1999B, 6: zur Kunstgeschichte nach Aufgaben als Verhältnis zwischen Angebot und Nachfrage. Cf. Stratmann 1998, 169: «Funktionsgeschichtliche Ansätze, Sammelbegriff für eine heterogene Gruppe von literaturwissenschaftlichen Strömungen der 60er und 70er Jahre mit kulturwissenschaftlich orientierten Perspektiven und Fragestellungen.» Cf. SCHNEIDER 1996: zum sozialgeschichtlichen Ansatz.

${ }^{15}$ Cf. Belting 1995, 98-99: «In allen diesen fällen ist Funktion, wie wir sagten, als ¿Umgang mit Bildern oder Bedarf nach solchen〉 verstanden, also aus der Sicht der Rezeptionshaltung des Publikums als eine sozialpsychologische Funktion. Rezeptionshaltung vor dem Bild ist aber mangels anderer Informationen oft nur aus den Bildern selber zu erfragen, weil sie qua Definition subjektive Züge trägt und sich nicht auf umgrenzte Situationen einschränken lässt. Davon zu unterscheiden ist jene Funktion, die nicht nur subjektive Erlebnisweise, sondern objektive Zweckbindung ist, also einen Anwendungsfall oder eine Spezialisierung der Bildfunktion darstellt. Diese applizierte Funktion erklärt sich vorrangig aus dem Kontext, dem sie zugeordnet ist.»

${ }^{16}$ BAKOš 2001. 
nerhalb seiner historischen Rahmenbedingungen hat jedenfalls in den letzten Jahrzehnten eine herausragende Rolle in den cultural studies eingenommen. ${ }^{17}$

Trotz seiner sehr verbreiteten Verwendung wurde der Funktionsbegriff in der Kunstwissenschaft kaum reflektiert. Wie Werner Busch zu Recht beobachtet, hat der Begriff «Funktion` auf methodischer Ebene «in der Geschichte des Faches Kunstgeschichte [...] überraschenderweise so gut wie keine Rolle gespielt». ${ }^{18}$ In der Tat wirft die Durchsicht kunstwissenschaftlicher Datenbanken eine Fülle von Einträgen zu konkreten Funktionen von Einzelwerken aus, weist allerdings auf keine methodologische Diskussion des Begriffs hin. Dass funktionalistische und utilitaristische Fragestellungen in der Selbstreflexion der heutigen Kunstwissenschaft im Gegensatz zur Soziologie und Philosophie - verhältnismäßig geringe Beachtung finden, mag daran liegen, dass die gesellschaftliche und zivilisatorische Relevanz von Kunst wissenschaftspolitisch wenig wahrgenommen wird, wie auch daran, dass Kunstwissenschaft weiterhin am zweifelhaften akademischen Ideal künstlerischer Zweckfreiheit festzuhalten pflegt, statt sich mit kulturwissenschaftlichen und -politischen Relevanzfragen zu konfrontieren. ${ }^{19}$

\section{$\langle$ Kontext〉}

Der kunst- und kulturgeschichtliche Begriff «Kontext> ließe sich, mit Rückgriff auf die Literaturtheorie, als die Menge der für die Erklärung eines Kunstwerkes, Bildes oder Gegenstandes relevanten Bezüge definie-

${ }^{17}$ Cf. Belting 1986, Zijlmans / Halbertsma 1995, Gérard Mermoz in TURNer 1996, vol. 2, 535-540 (contextualism, culture as context), Busch 1997в und Baumgartner 1998, 156-158: zur Kunst im Kontext. Cf. Busch 1987, 3: Die Frage nach der Funktion der Kunst sei in der Lage, «das Verhältnis zu erhellen, das zwischen den jeweiligen historischen Rahmenbedingungen der Kunst und ihrer individuellen Erscheinung besteht.» Cf. BöHME ET AL. 2000, 13: zur Kontextualisierung in den cultural studies. Cf. Collins 1990, 322: «But for now, at least, art history seems increasingly synonymous with the contextual study of art - however that might be.» Cf. KEMP 1991: «Die gängige Orientierungsgröße der Kunstgeschichte in Forschung und Praxis ist das Einzelobjekt [...]: Unter dem weiten Titel «Kontextualismus〉 sind seit Jahren viele Bestrebungen im Gange, [...] das Kunstwerk aus seinen historischen, sozialen, psychologischen, literarischen, kulturgeschichtlichen Verflechtungen» zu erklären; dieser Ansatz habe sich «durchgesetzt» und müsse «also nicht mehr auf sein Innovationspotential hin getestet werden.» Ib., 98: «Wie jede Äußerung kann Kunst ohne Kontext nicht gedacht werden.»

${ }_{18}$ Busch 1997в, 24: «Im Grunde genommen genügt ein Satz: In der Geschichte des Faches Kunstgeschichte hat der Begriff überraschenderweise so gut wie keine Rolle gespielt.» Cf. Didi-HuBERMAN 1996, 61: «Mais le mot 〈fonction〉 [...] est bien peu souvent interrogé dans ses usages méthodologiques ou heuristiques.»

${ }^{19}$ Cf. EMmet 1967; Malinowski 1988; Steinbacher / ReichenHALl 1990; MenGET 1999: zum soziologischen Funktionsbegriff. Cf. SteINER ET AL. 1971-1995 und GANSLANDT 1980-1996: zum philosophischen Funktionsbegriff. Cf. KLOTz 1995, 41-42: zur Relevanzfrage. ren. ${ }^{20}$ Unter 〈Kunst im Kontext〉 wird spezifischer eine kulturgeschichtlich und interdisziplinär ausgerichtete Untersuchung auch nicht künstlerischer Produktionsund Rezeptionsbedingungen von Bildwerken verstanden. ${ }^{21}$ Sie will sich einerseits von einer tendenziell ahistorischen Stil- und Formanalyse oder kennerschaftlichen und psychologisierenden Kunstbetrachtung absetzen, wie sie etwa von Alois Riegl (1858-1905) oder Heinrich Wölfflin (1864-1945) initiiert worden ist, als auch andererseits gegen die darauffolgende inhaltzentrierte und textorientierte Ikonologie, wie sie Erwin Panofsky (1892-1968) vertreten hat. ${ }^{22}$

Die Funktionsgeschichte ist Teil der Kontextforschung, insofern eine funktionale Relation den Kontext erst herstellt. ${ }^{23}$ Die funktionsgeschichtliche Frage «wo$\mathrm{zu}$ ? > ist offener und allgemeiner als das, was den Kontext der Entstehung und Wirkung von Kunstwerken gemeinhin zusammenhält, wie etwa die Verben sbeeinflus-

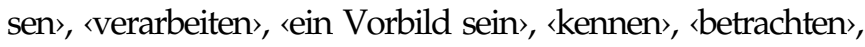
〈repräsentieren〉, indoktrinieren〉, ‘spiegeln〉, ‘konstruieren〉, ‘an einem Diskurs teilhaben〉, ssymbolisieren〉 und dergleichen mehr. ${ }^{24}$ Diese Begriffe objektivieren in der Regel die Wunschvorstellung einer kausalen Relation, deren konkrete Wirklichkeit schwer zu belegen ist und die leichter in einer abstrakten und allgemeinen Kategorie aufzuheben ist. ${ }^{25}$ Die Frage nach der Funktion, nach dem Gebrauch, nach der Kausalität, die das Kunstwerk mit seiner Umwelt zu einem bedeutungsvollen Kontext verknüpfen, wäre immer wieder in aller Schärfe zu stellen, um den kunsthistorischen Wunsch zu zügeln, die projizierte Schlüssigkeit der Deutung in der Vergangenheit vergegenständlicht zu sehen.

\section{Kulturgeschichte}

Die kunstwissenschaftliche Funktionsgeschichte versucht, Form und Inhalt auf Grund einer Zweckrelation miteinander zu verknüpfen. Deswegen sieht Werner Busch, in Anlehnung an Horst W. Janson (1913-1982), im funktionsgeschichtlichen und kontextuellen Ansatz eine Synthese aus formgebundener Stilgeschichte und inhaltgebender Ikonologie in Richtung einer allgemeinen, bildorientierten Kulturgeschichte. ${ }^{26}$ Die Funktions-

${ }^{20}$ Cf. Danneberg 1997-2000, 333: «[Der Kontext ist] die Menge der für die Erklärung eines Textes relevanten Bezüge.»

${ }^{21}$ Cf. Hirdina 2000-2001, 588: «Als Begriffe der Ästhetik werden 〈Funktionalismus〉, ‘funktionalistisch〉, ffunktional〉 (seltener ‘funktionell`) auf außerkünstlerisches Gestalten bezogen.»

${ }^{22}$ Cf. BAuer 1976, 74-81: Für Riegl sei der Gebrauchszweck ein Hindernis für das Kunstwollen. Ib., 93-99: zur Ikonologie.

${ }^{23}$ Cf. DANNEBERG 1997-2000, 333: 〈Kontext〉 sei ein «relationaler Ausdruck».

${ }^{24}$ Cf. z. B. BAXANDALl 1985, 58-62, cap. Excursus agaist influence.

${ }^{25}$ Cf. Zijlmans / HalberTsma 1995, 28: «die Beziehung zwischen Kunstwerk und Kontext letztlich ungeklärt». 
und Kontextgeschichte, welche die Untersuchungsgegenstände in ihrem jeweiligen Lebenszusammenhang zu betrachten bemüht ist, hat sich in der Nachkriegszeit in der westlichen Forschung etabliert. In der zweiten Jahrhunderthälfte dürften kulturhistorische Phänomene, wie etwa unter anderen die Abwendung von einheitlichen oder totalitären Denkmodellen, die Hinwendung zu kulturrelativen und polyfokalen, soziologischen, linguistischen, strukturalistischen und anthropologischen Betrachtungsweisen, die Analyse der expandierenden Alltagskultur, die Rezeption des marktwirtschaftlichen Nutz- und Leistungsdenkens und dergleichen mehr, den heutigen kunstwissenschaftlichen Funktionsbegriff geprägt haben. ${ }^{27}$

Auf der theoretischen Ebene ermöglicht die Funktionsgeschichte auf Grund ihrer umfassenden und interdisziplinären Fragestellung eine stärkere Anbindung an andere, beispielsweise sozialwissenschaftliche oder soziobiologische Disziplinen, welche mit einem funktionalistischen Denken vertrauter sind; mit ihnen wäre auch ein Instrumentarium zu erarbeiten, das anthropologische Grundmuster des Bildgebrauchs von anderen Arten der Objektnutzung zu differenzieren hilft. ${ }^{28}$ Die

${ }^{26}$ JANSON 1982, 36: «The great promise of Burckhardt's functional approach is that it enables us to see style and iconography under a common perspective instead of keeping them in separate and mutually exclusive mental compartments.» Busch 1987, 7: «Der Funktionswandel des Kunstwerkes greift grundsätzlich in seine Struktur ein, betrifft Form und Inhalt, vor allem aber das Verhältnis von Form und Inhalt.» Busch 1997в, 25: «Grob vereinfachend kann man sagen: Einer langen Phase überwiegend formgeschichtlicher Fragestellungen folgte eine lange Phase überwiegend inhaltlich-themengeschichtlicher Beschäftigung mit der Kunst. Heute wird von den verschiedensten Seiten her der Versuch einer Synthese von formund inhaltsgeschichtlicher Betrachtungsweise versucht. Als ein solcher Versuch mag auch der hier vorgeschlagene funktionsgeschichtliche Ansatz begriffen werden.» Cf. Belting 1995, 22: «Zwischen der Struktur der Bildsprache und der Komplexität ihrer semantischen Inhalte besteht ein Zusammenhang. Die Folge ist die wachsende Autonomie der bildsprachlichen Form als 〈Kunst $\rangle$. Die Forschung hat diesen Zusammenhang wenig untersucht, weil sie alternativ auf Stilkritik oder Ikonografie festgelegt war. Im einen Fall wurde im Werk der Stil, im anderen Fall der 〈Inhalt〉 als religions- oder kulturgeschichtliches Produkt isoliert. Dagegen blieb das Bild oft unbefragt in seiner Eigenschaft als visueller Text, der Inhalte und Funktionen auf je spezifische Weise in Form umsetzt.» Cf. Suckale 1995, 9: «Mit der Funktions-, Sozial- und Rezeptionsgeschichte, der Bedeutungsforschung und anderen mehr weitet sich unser Fach zu einer umgewandelten Kulturgeschichte.» Cf. SEDLMAYR 1958, 11.

${ }^{27}$ Cf. Stratmann 1998, 169: «Das Aufkommen solcher funktionsgeschichtlicher Ansätze war als doppelte Reaktion auf die literaturtheoretischen Trends der damaligen Zeit [1960er und '70er Jahre] zu verstehen: auf die rein intrinsischen Ansätze wie der new criticism einerseits sowie die Literatursoziologie und die marxistische Literaturtheorie andererseits. Während man den ersteren die Ausblendung der historischen Dimension und des kulturellen Kontextes von Literatur vorwarf, erschienen die letzteren auf Grund ihrer ideologisch voreingenommenen und wegen ihres systematischen Charakters unflexibel.» Cf. JeHLE 2000-2001: Alltagskultur.
Funktionsgeschichte verspricht, die postmoderne Mehransichtigkeit der Dinge in die Vergangenheit auszudehnen und zugleich die Frage nach Zweck und Sinn sowohl der Kunst wie auch der kunsthistorischen Untersuchung nicht aus den Augen zu verlieren. ${ }^{29}$

\section{Methode?}

Die Fundierung einer funktionsgeschichtlichen Methode, sollte es eine solche geben, kann hier nicht geleistet werden. Sie soll bloß skizzenhaft kartiert und zur Diskussion gestellt werden. Denn vorab wäre zu klären, welche Fragen eine solche Methodendiskussion zu beantworten hätte, und ob sich daraus begriffliche Komplikationen, Scheinprobleme und sprachliche «Verhexungen $>$ erst ergeben würden. ${ }^{30}$ Es soll hier eher darum gehen, den scheinbar selbstverständlichen Begriff ¿Funktion` zu hinterfragen und ihn in das kritische Bewusstsein zu rücken, um ihn schließlich auf pragmatische Weise seiner - bestenfalls nun verunsicherten Selbstverständlichkeit zurückzuführen. Zwei konzeptuelle Schwierigkeiten treten jedenfalls zu Tage, welche die Begriffe «Methode` und ‘Funktion〉 betreffen und welche im Folgenden kurz zu umreißen sind.

Möchte man, erstens, unter dem Begriff «Methode` ein kohärentes, logisch oder mathematisch fundiertes, gänzlich nachvollziehbares Begriffs- und Operationssystem verstehen, so kann die kunsthistorische Funktionsanalyse, die ihre lose verbundenen Begriffe wie etwa 〈Andacht> oder ‘Information` aus disparaten Wissens- und Erfahrungsgebieten bezieht, keine Denkmethode im engeren Sinne des Wortes darstellen. ${ }^{31}$ Dieses unüberwindbare Problem teilt sie mit den anderen Geisteswissenschaften, wenn diese sich anhand eines me-

${ }^{28}$ Cf. z. B. Rump 1993 und Eibl-Eibesfeld / Sütterlin 1992: zu Kunst und Verhaltensforschung. Cf. z. B. BAUER 1976, 152-155 und BeLting 2001: zur Kunstanthropologie.

${ }^{29}$ Auf der praktischen Ebene hingegen dürfte die Funktionsgeschichte beispielsweise eine ganzheitlichere und behutsamere sogenannte «Ensembledenkmalpflege〉 oder etwa eine didaktischere und lebensnahe Museologie vorangetrieben haben. Cf. z. B. HuSE 1996, 211-215: zur interdisziplinären Ensembledenkmalpflege. Cf. KEMP 1991, 99: Das Ensemblebedürfnis sei ein Resultat kunsthistorischer und musealer Dekontextualisierung. Cf. MAттICK 1996, 70-71: Die Dekontextualisierung und Musealisierung von Kunstwerken als eine Folge des napoleonischen Kunstraubs sei bereits von Antoine Chrysostôme Quatremère de Quincy beklagt worden. Cf. KleimanN / SchmücKer 2001, 8: «Wer nach der Funktion von Kunst fragt, fragt mithin immer auch danach, was Kunst denn eigentlich ist.»

${ }^{30}$ Cf. DANIEL 2001, 401: «[Es ist zu bemerken,] dass Theorie- und Methodendebatten eine ihnen eigentümliche Tendenz haben, Begriffe und Verfahrensweisen der alltäglichen und der wissenschaftlichen Praxis in solche zu verwandeln, die im Zusammenhang ihrer jeweiligen Praxis selbst nicht mehr reflektierbar zu sein scheinen». Cf. LuHMANN 1998, 222-224.

${ }^{31}$ Cf. ZAUnschirm 1975, 3-22: zur Kunstwissenschaftstheorie. 
thodologischen Gegensatzes zu den Naturwissenschaften definieren wollen. Es handelt sich eher um einen methodos, im Sinne einer Wegbeschreibung oder eines Wegweisers, um eine retrospektive, rekonstruierende, vereinfachende, einschränkende, meistens apologetische und das sprechende Ich verheimlichende Darstellung eines induktiven Gedankenverlaufs, um eine biografische Forschungserfahrung. ${ }^{32}$

Die nachvollziehbare Darstellung der jeweiligen Vorgehensweise in der kunstwissenschaftlichen Literatur ist selten auf einer a priori konzipierten und streng applizierten Strategie begründet, sondern ergibt sich zumeist aus dem a posteriori erfahrenen, nachgeahmten oder intuitiven Suchen selbst. So gesehen macht eher der Erfolg die Methode aus und weniger die Methode den Erfolg. ${ }^{33}$ Unter anderen Teilgebieten der Kunstwissenschaft gehört daher auch die Funktionsgeschichte zur nicht näher definierten Kategorie der so genannten 〈Forschungsansätze〉, ‘Fragestellungen〉 und 〈Betrachtungsweisen〉, die mittels Begriffskonstellationen, Argumentationsschemata und einer narrativen Zusammenstellung von Daten einen Sachverhalt in einem bestimmten, für eine Forschungsgemeinschaft interessanten Blickwinkel vorstellig machen oder evozieren möchten, über dessen Relevanz und Ertrag jeweils zu debattieren ist. ${ }^{34}$

Zweitens bezeichnet der Begriff ‘Funktion〉 keine gegenständliche Qualität, sondern als Vorstellung und Ausdruck, dessen Gebrauchsweise zu untersuchen wäre, eine verallgemeinerte und abstrakte Form einer Verrichtung, die Möglichkeit einer Handlung oder Benutzung, eine reine Zweckbestimmung, die vom Gegen-

\footnotetext{
32 Cf. GinZburg 1988, 12: «Die beiden Dinge, der Gang der Forschung und die Diskussion über die Methode, sind doch miteinander verbunden. [...] Man muss von beidem erzählen: von der Forschung und von den Ergebnissen. Die Methode, sagt Granet, ist etymologisch gesehen - einfach der Weg, den man einmal eingeschlagen hat.» Cf. Ginzburg 1993, 113: «alle Phasen, die eine Forschung durchläuft, [sind] konstruiert und nicht gegeben». Cf. JoHNSON 1988, 6: «It is usually to begin with rather too restricted an idea for the development of a topic; once it is realized that the topic leads in all directions, these different courses must be followed up without losing sight of the original goal. Then one compresses the richness and texture of the research into the topic as it has come to be.»

${ }^{33}$ Cf. DANIEL 2001, 14: «Methode und Ergebnis, so lautet ein zentrales kulturwissenschaftliches Credo, stehen in einem zirkulären Verhältnis zueinander: Sie bedingen sich gegenseitig.»

${ }^{34}$ Cf. DANIEL 2001, 12: «Es gibt kein primär Gegebenes für die Kulturgeschichte, es gibt nur - um es mit dem amerikanischen Philosophen John Dewey zu sagen - das Genommene, also die Auswahl und Gestaltung von Themen unter bestimmten Fragestellungen.» Cf. ib., 430-443: zu Sprache und Narrativität. Cf. STRATMANN 1998: zur Literaturtheorie. GANSLANDT 1980-1996, 695: «Trotz aller Kritik hat sich der strukturell-funktionale Ansatz, der mehr Forschungsprogramm als methodische Forschung ist, in der Soziologie durchgesetzt.» Cf. VeYne 1996, 9: «Non, l'histoire n'a pas de méthode: demandez donc un peu qu'on vous montre cette méthode». Cf. ib., 10: «l'histoire est un roman vrai».
}

stand weg auf andere Gegenstände und auf eine Tätigkeit verweist. ${ }^{35}$ Die aristotelische und scholastische Vorstellung von Akt und Potenz als Erklärungsmodell für Bewegung und Entwicklung, wie sie beispielsweise in Johann Wolfgang von Goethes (17491832) Definition - «Funktion, recht begriffen, ist das Dasein in Tätigkeit gedacht» - zum Ausdruck kommt, würde in einem kulturhistorischen Kontext nicht greifen, weil sie apriorische Möglichkeitsstrukturen und Zwecke voraussetzt und in das Wesen des Gegenstandes projiziert, die erst zu entdecken sind. ${ }^{36}$

\section{Eigenschaft?}

Ein Gegenstand 〈hat〉 weniger Funktionen, als 〈man〉 viel mehr mit ihm <etwas tun kann. Indem das Ding 〈als〉 Mittel zu einem Handlungsziel (fungiert), ist es mehr als es selbst und spielt eine 〈Rolle` innerhalb einer anthropozentrischen oder als anthropomorph vorgestellten Handlung. Eine Funktion setzt ein gezielt handelndes und denkendes Subjekt voraus, das sich der Dinge und seiner eigenen Physis als Werkzeuge bedient. Funktionen kommen eher Lebewesen und menschlichen Erzeugnissen als toten Naturdingen zu. Eine Sense, ein Nashornkäfer oder eine Tulpenblüte können als das Produkt und Bild eines biologischen und kulturellen Selektionsprozesses gesehen werden, der einen einzelnen Lebenswillen voraussetzt. Die Funktion ist vielmehr in den Intentionen eines handelnden Subjekts zu verorten als im Gegenstand selbst. ${ }^{37}$ Ein Atheist kann nicht sinnvoll von der Erderwärmung als einer Funktion der Sonne sprechen. Folgerichtig und bezeichnenderweise verweist die Encyclopédie, welche die physiologische Funktion als Handlung definiert, zu welcher die Organe auf Grund ihrer Struktur bestimmt sind, auf die Vorstellung der Welt als zweckgerichtete und von der christlichen Gottheit entworfene Maschine und damit auf den theologischen, mechanistischen und organizistischen Hintergrund des Funktionsbegriffs. ${ }^{38}$

${ }^{35}$ Cf. Janson 1982, 4 und Baschet 1996, 17: Der Funktionsbegriff sei schwer zu definieren. Cf. BRocKard 1973 und ThiEl 1973. Cf. SCHмÜCKER 2001, 21: «der Funktionsbegriff zielt auf die potentielle Dienlichkeit einer Sache».

${ }^{36}$ SCHAdewald 1978-1994, vol. 4, 1025: Goethe. Cf. SCHLÜTER 1971-1995: zu Akt und Potenz.

${ }^{37}$ Cf. BätschmanN 1992, 66-68: zur Intention.

${ }^{38}$ Diderot / D'Alembert 2000, vol. 7, 51 a, Fonction: «Fonction (Économie animale): est une action correspondante à la destination de l'organe qui l'exécute. [...] Comme on a reconnu de tout tems, qu'un être infiniment sage est l'auteur de notre corps \& de ses divers organes ; on a aussi senti qu'il avoit arrangé \& disposé toutes les pieces de cette admirable machine, selon des vûes ou des destinations: \& c'est pour remplir ces vûes qu'elles agissent ; en conséquence de quoi, on appelle fonctions ces actions, comme étant faites pour s'acquiter d'un devoir auquel leur structure \& leur position les engagent.» Cf. Busch 1987, 4. 
Das Definitionsproblem würde bloß auf eine heiklere ideelle Ebene verschoben, wenn Funktionen als Eigenschaften nicht der Kunstwerke, sondern der Kunst 〈an sich〉 vorgestellt würden. ${ }^{39}$ Obwohl Funktionen sich nicht als materielle, messbare Qualitäten manifestieren, sondern eher dem Denken, Sprechen, Handeln des Menschen eigen sind, gehören funktionale Spuren an Objekten zu den Indizien, die - aus einer unabdingbaren anthropologischen Perspektive gesehen - auf eine bestimmte Intention oder Rezeption schließen lassen. ${ }^{40}$ Die Nutzung oder Zweckmäßigkeit eines Gegenstandes drückt sich als eine uneindeutige Form aus, beispielsweise als Abnutzungsspur oder Ergonomie, die in einem Lebens- und Handlungszusammenhang erprobt oder vorgestellt werden muss, um für den Betrachter innerhalb seines Erfahrungshorizonts zu einem Funktionszeichen zu werden. Beim funktionalen Kontext handelt es sich eher um ein von Menschen gesponnenes Handlungs- und Bedeutungsgewebe als um ein bloßes Kausalitätsgefüge, wie dies Clifford Geertz für die Ethnologie formuliert hat. ${ }^{41}$ In dieser Hinsicht erscheint eine Funktionsbestimmung nicht als ein ästhetisches, sondern als ein wertendes, interpretierendes und teleologisches Urteil..$^{42}$ Einer methodischen Fundierung der kunst- und kulturhistorischen Funktionsanalyse käme die Sprachphilosophie Ludwig Wittgensteins insofern entgegen, als sie Bedeutung vor allem durch und im Sprachgebrauch begründet, der selbst in einer weiter gefassten, historischen und kulturrelativen «Lebensform» eingebettet ist - was 〈Kunst〉 und ihre spezifischen Funktionen seien, lässt sich nur von Fall für Fall kulturgeschichtlich beschreiben. ${ }^{43}$

Aus dieser Problemskizze lässt sich erahnen, vor welche sprachliche und begriffliche Schwierigkeiten die gegenstandsorientierte Kunstwissenschaft gestellt würde, sollte sie den Terminus «Funktionsgeschichte» methodisch präziser fassen wollen. Beispielsweise

${ }^{39}$ Cf. SCHмüCKER 2001, 17: «Es gibt also Funktionen, die ein Kunstwerk als Kunstwerk besitzen kann und die sich nicht dem physischen Objekt zuschreiben lassen, in dem sich das fragliche Kunstwerk manifestiert.» Cf. ib., 25: Der Kunstbegriff sei wertend, und keine hinreichendes kunstästhetisches Kriterium zur Bestimmung eines Kunstwerkes als solches gebe es nicht. Kunstwerke lassen sich nur deskriptiv auf Grund eines gesellschaftlichen, sich wandelnden Konsenses als solche ausmachen.

${ }^{40}$ Cf. Ginzburg 1986: zur Geschichtswissenschaft als Spurensicherung.

${ }^{41}$ GeErtz 1987A, 9: «dass der Mensch ein Wesen ist, das in selbstgesponnene Bedeutungsgewebe verstrickt ist, wobei ich Kultur als dieses Gewebe ansehe. Ihre [ethnologische] Untersuchung ist daher keine experimentelle Wissenschaft, die nach Gesetzen sucht, sondern eine interpretierende, die nach Bedeutungen sucht».

${ }^{42}$ Cf. De Zurko 1957в, 3: «Functionalism is [...] a value.» Cf. DidiHuBERMAN 1996, 76: zum teleologischen Urteil.

${ }^{43}$ WitTgenstein 1984, 250, no. 23: «Das Wort «Sprachspiel〉 soll hier hervorheben, dass das Sprechen der Sprache ein Teil ist einer Tätigkeit, oder einer Lebensform.» Cf. MAJETSCHAK 1998, 826: zur Klärung ästhetischer Begriffe durch die Beschreibung der Sprachspiele innerhalb einer epochen- und kulturbedingten Lebensweise. C. GeErTz 1987в, 25. müssten Funktions- und Handlungstypen als solche definiert, Kategorien der Nutzungsintention und -rezeption festgestellt, Fragen der Zweckmäßigkeit und Kausalität geklärt werden. Alleine schon die moderne Debatte um den architektonischen functionalism lehrt, dass das, was nach Funktionalität aussieht oder klingt, noch lange nicht realiter funktional oder auch nur rational begründet sein muss. ${ }^{44}$ Dennoch sollen einige Vorzüge und Grenzen eines kunsthistorischen Funktionsbegriffs im Folgenden angeführt werden, um mit ihm kritisch operieren zu können.

\section{Polyfunktionalität}

Wie ‘funktionieren〉 Kunstwerke? Weil dem Wort «Funktion〉 mechanistische Konnotationen anhaften, möchte man zunächst intuitiv die Aura <freier` oder ‘autonomer> Kunst davon verschonen - als hätte es beispielsweise keine Maschinenästhetik der Moderne gegeben. ${ }^{45}$ Auch die funktionsgeschichtliche Forschung scheint sich darüber einig zu sein, dass Kunstwerke nicht wie Maschinen funktionieren und sich nicht mittels naturwissenschaftlicher Kausalität erklären lassen.

In der Tat wird der im engeren Sinne technische Begriff «Funktion` der relativ offenen Nutzbarkeit und der Multifunktionalität von Kunstobjekten und Bildern nicht gerecht. Diese lassen sich nicht durch die klar definierbare, monokausale und reine Funktionalität eines Werkzeugs charakterisieren, wie etwa Ernst H. Gombrich (1909-2001) bemerkt. ${ }^{46}$ Ein rein funktionalistisches Erklärungsmuster ist unzureichend: So kann man beispielsweise die Erscheinungsform von Gianlorenzo Berninis (1598-1680) Altarbaldachin von Sankt Peter nicht aus seiner sanktionierten, rein technischen Funktion als Altarschirm gegen herabfallenden Taubenschmutz ableiten. ${ }^{47}$ In diesem Sinne plädiert auch Erik Forssman für eine «Multipelkausalität» historischer Ereignisse, beziehungsweise für eine Multifunktionalität der Kunstgegenstände, die sich von der Monokausalität der Naturwissenschaften unterscheidet. ${ }^{48}$

${ }^{44}$ Cf. De Zurкo 1957в, 4: «Functionalist trends are those which stress the importance of fitness and utility. Functionalism may or may not involve a theory of beauty.» Ib., 8-9: Der architektonische Funktionalismus der Moderne sei kaum auf Rationalität, sondern auf mechanischen, organischen oder moralischethischen Analogien begründet. Cf. Posener 1977. Cf. Jones 1996: Literaturhinweise.

${ }^{45}$ Cf. KLEIMANN / SCHMÜCKER 2001, 7: Obwohl die Kunsttheorie zumeist eine «teleologisch-funktionalistische Terminologie» vermeidet, handelt es sich um die Funktion der Kunst, wenn man nach Wert, Sinn, Aufgabe, Ziel der Kunst fragt.

${ }^{46}$ Gombrich 1999A, 49: «The techniques of image-making never serve the same kind of statable purpose as does the technology of aviation or medicine.»

${ }^{47}$ Cf. BRAUN 1924, 185-187: zur funktionalen Definition von Altarbaldachinen. 
Nach Jérôme Baschet ist der Bedeutung und Wirkung von Kunstgegenständen ein Funktionsüberschuss oder eine Multifunktionalität eigen, eine Art Benutzungsfreiheit und Bedeutungsspielraum..$^{49}$ Er warnt dabei vor einer unkritischen Anwendung eines mechanistischen, von Émile Durkheim (1858-1917) geprägten, soziologischen Funktionalismus. Dem Begriff «Funktion` eile gar ein schlechter Ruf voraus, weil er die Einschränkung des Blickwinkels auf einen gesellschaftlichen Mechanismus evoziere. Bedeutung lasse sich nicht allein auf Funktionen reduzieren; kein Bild oder Kunstwerk könne durch eine einzige Zweckbestimmung erklärt werden, etwa das konkrete religiöse Tafelbild durch die bloße Andacht. Daher müsse man bei Kunst zumindest von «Funktionen` im Plural sprechen. ${ }^{50}$

Während man der Feststellung zustimmen mag, dass die Form von Kunstwerken - und von Dingen überhaupt - sich nicht kausal aus vorgegebenen Funktionen, die keine notwendigen Gestaltungs- und Existenzbedingungen sind, ableiten lässt, wird hingegen kaum in Abrede zu stellen sein, dass wahrscheinliche Funktionen zum nachträglichen Verständnis der Dinge beitragen können. ${ }^{51}$

\section{Mechanizismus?}

Im Unterschied zu Maschinen verrichten Kunstwerke ihren Dienst nicht automatisch und zielgerichtet; sie gehen nicht in einer einzigen, eng definierten Funktion als ihrem alleinigen Daseinsgrund auf, deren Verlust ihre Verschrottung bedeuteten würde; sie werden nicht nach ihrer messbaren Leistung oder Effizienz beurteilt; und sie sind in der Regel auch ohne Gebrauchsanweisung genießbar. Zwar steht auch das Altarretabel in einem funktionalen Gefüge, in einem liturgischen Raum, und seine Funktion und Bedeutung kann sich in einem

\footnotetext{
${ }^{48}$ Forssman 1974, 50: «Ursachen sind für uns alle Ereignisse biographischer, psychischer, sozialer und politischer Art, die im Sinne von Bedingungen (das heißt vorwiegend einschränkend) oder von Gelegenheiten (das heißt vorwiegend fördernd) auf das Werden und das Sosein von Kunstwerken einwirken. Wie in der Geschichte, haben wir es auch in der Kunstgeschichte immer und in jedem einzelnen Falle mit einer Multipelkausalität zu tun, das heißt jeder Versuch, es der Naturwissenschaft gleichzutun und zu einer Monokausalität im Sinne einer einzigen oder letzten Ursache überzugehen, ist zum Scheitern verurteilt. [...] Nur mit der entgegengesetzten Einstellung [zum Strukturalismus] nähert man sich der geschichtlichen Wirklichkeit des Kunstwerks: Um auch nur eine begrenzte Anzahl seiner zentralen Eigenschaften annähernd erklären zu können, müssen wir einer Unzahl von Ursachen nachgehen.»

${ }^{49}$ BASCHET 1996, 16: «L'œuvre, par son sens et ses effets, déborde toujours sa fonction.» Cf. Didi-HubERman 1996, 83.

${ }^{50}$ BASCHET 1996, 15: «Le mot <fonction» a mauvaise réputation; il évoque une vision étroite». Ib.: Funktion und Bedeutung seien nicht mechanistisch auf Eines reduzierbar. Ib., 16: zu〈Funktionen〉 im Plural. Ib., 21: zur Andacht. Cf. SHEARMAN 1972A, 369.

${ }^{51}$ Cf. DANIEL 2001, 400-409: zu Erklären und Verstehen.
}

anderen Zusammenhang, etwa dem Museum, entsprechend ändern, doch nicht so drastisch wie beim Zahnrad ohne Uhrwerk und dem Zeiger ohne Zifferblatt. Ebenso wenig erscheint die Kunstproduktion als eine mechanische Erzeugung funktionaler Gegenstände: Der Künstler malt ein Bild nicht so wie der Müller das Korn. ${ }^{52}$ So gesehen erschiene ein Kunstwerk auf komplexere Weise in einen sozialen Kontext eingebunden als ein Maschinenteil im Räderwerk und es würde in dieser Hinsicht einem Lebewesen ähneln, das in seinem Biotop mannigfaltige, rückgekoppelte und sich verändernde Funktionen erfüllt. ${ }^{53}$

Kunst und Technik sind jedoch keine Gegensätze. Es ist zu bedenken, dass es sich bei einer solchen Differenzierung des Funktionsbegriffs nur um graduelle Unterschiede und Ähnlichkeiten zwischen Kunst und Technik, Kunstwerk und Werkzeug, handeln kann. So ist etwa ein römischer Aquädukt ein Produkt aus Technik und Kunst, und ein Renaissancearchitekt hätte selbst kaum zwischen Baukunst und Bautechnik unterschieden. ${ }^{54}$ Eine funktionalistische Betrachtungsweise ist jedenfalls eher bereit zu akzeptieren, dass Technologie eine wesentliche Rolle in Kunst und Kultur spielt. ${ }^{55}$

$\mathrm{Ob}$ man den technischen Funktionsbegriff in der Kunstwissenschaft zulassen will, wird unter anderem davon abhängen, ob der unscharfe und möglicherweise obsolete Begriff 〈Kunst〉 den Blick auf eine Gruppe nicht ästhetischer Funktionen versperrt, in welche 〈Kunst〉 sich eventuell auflösen ließe. ${ }^{56}$ Wie tief der an sich kurzsichtige technische Funktionsbegriff in die vielschichtige Ästhetik hinabblicken kann, hängt davon ab, in wie viele, verfeinerte Unterkategorien man ihn potenzieren möchte, so dass er auch komplexe Kunstphänomene erfassen kann, wie beispielsweise das so genannte «Schöne), das so auf nicht ästhetische Wirkungen reduziert würde. Die Extremposition, jegliche Kausalität und Bedeutung als eine Funktion verstehen zu wollen, birgt jedoch die Gefahr, den Funktionsbegriff so sehr auszuweiten, dass seine Aussagekraft entsprechend geschwächt und die Argumentation lediglich auf eine mechanistische Metaebene verschoben wird, auf welcher die Welt der Phänomene zu einer gigantischen Billardpartie zugleich simplifiziert wie auch verkompliziert wird.

${ }^{52}$ Cf. Gombrich 1963, 111: «Symbols do not carry meaning as trucks carry coal.»

${ }^{53}$ Cf. KeMP 1991, 94-96.

${ }^{54}$ Cf. Moos 1974, 209-210: zum Renaissancezweckbau.

${ }^{55}$ Cf. De Zurкo 1957в, 240: «Functionalism is the only aesthetic which frankly accepts the world of technology as an important part of the pattern of culture.»

${ }^{56}$ Cf. BAUER 1976, 14-17: zum Begriff 〈Kunstwerk〉 als Gegenstand der Kunstwissenschaft. 


\section{Autonomie?}

Das kunsthistorische und -theoretische Missbehagen gegenüber einem mechanistischen Funktionsbegriff entspringt vermutlich dem Wunsch, ein geistiges Reservat <autonomer〉 oder 〈freier〉 Kunst vor zersetzender interdisziplinärer Analyse zu schützen, welche dem Fach den exklusiven Zugriff auf ihren Gegenstandsbereich, durch den sich die Kunstwissenschaft definieren will, strittig machen würde. ${ }^{57}$ Wie Reinold Schmücker darlegt, hält vor allem die aufklärerisch und idealistisch geprägte Kunstphilosophie im deutschen Sprachraum, sei sie normativ oder deskriptiv, am Dogma der Kunstautonomie fest, auch wenn sie dem Kunstwerk, unter anderem Namen, einen höheren Zweck zuschreibt, wie zum Beispiel bei Theodor Wiesengrund Adorno (19031969). ${ }^{58}$ Selbst Funktionshistoriker beharren auf einer exklusiven «ästhetischen», über die Sinne vermittelten Funktion, die Kunstwerke von allen anderen Gegenstandsklassen unterscheide.$^{59} \mathrm{Ob}$ es eine solche allgemeine, quasi objektive «ästhetische Funktion〉 geben kann, wo doch sinnliche Wahrnehmbarkeit ein Merkmal aller Gegenstände ist, wird nicht zuletzt von der modernen und zeitgenössischen Kunst radikal in Frage gestellt. Die Tatsache, dass der Kunstbegriff jedem beliebigem Gegenstand zugeschrieben werden kann und worden ist, zeigt, dass es zwecklos ist, Kunstwerke <als solche», nämlich auf Grund einer zeit- und raumlosen ästhetischen oder künstlerischen Funktion definieren zu wollen.

Im Gegenteil: Die Funktionsgeschichte steht dem Ästhetischen nicht entgegen. Eine Ausdifferenzierung der Funktionstypen birgt nicht die Gefahr, das ominöse 〈Ästhetische〉 und «Künstlerische〉 aus der Kunstwissenschaft zu verbannen, sondern erlaubt umgekehrt die spezifisch künstlerischen und ästhetischen kontextuellen Aufgaben der Werke überhaupt erst klarer heraus-

${ }^{57}$ Cf. Kleimann / SCHMÜCKeR 2001, 7-8: «Aus dieser autonomieästhetischen Perspektive verfehlt die Frage nach der außerästhetischen Funktionen die Kunst. Umso bemerkenswerter ist, dass nicht wenige Kunsttheoretiker einerseits zwar die Autonomie der Kunst bejahen, andererseits aber der Kunst zugleich eine Funktion zuerkennen - auch wenn sie sich dazu eines anderen Begriffs bedienen». Cf. ib., 19: «Der kunstästhetische Antifunktionalismus ist unbegründet.»

${ }^{58}$ SCHMÜCKer 2001. Adorno 1998.

${ }^{59} \mathrm{Cf}$. BusCH 1987, 5: «Funktionsanforderungen werden zwar von außen an die Kunst herangetragen, bleiben ihr aber nicht äußerlich.» Ib.: «Jedes Kunstwerk ist zuallererst ästhetischer Natur: es wirkt sinnlich, es transportiert seine Mitteilung über die Sinne. Alle anderen Funktionen des Kunstwerkes kommen von außen - die ästhetische ist ihm eigen.» Ib., 7: «Das Kunstwerk ist grundsätzlich ästhetischer Natur, seine ästhetische Funktion kann jedoch Selbstzweck werden. Jedes Kunstwerk vermittelt seinen Sinn ästhetisch, es wirkt sinnlich. Diese grundsätzliche Wirkweise definiert das Kunstwerk als solches.» Cf. SCHMÜCKER 2001, 26: «Konstitutiv für Kunst ist ihre kunstästhetische Funktion, d. h. die Funktion, eine ästhetische Erfahrung hervorzurufen, die in ein Verstehen einmünden kann und will.» zuarbeiten. ${ }^{60}$ Durch eine Analyse der ausdrücklich nicht künstlerischen Funktionen, wie sie für diesen Ansatz charakteristisch ist, treten die historischen ästhetischen Aufgaben oder Autonomiemerkmale der Kunst, beispielsweise der Galeriekunst, erst deutlich zu Tage. ${ }^{61}$ Jan Mukarovský (1891-1975) etwa begreift die ästhetische, nicht praktische Funktion von Gegenständen, also deren Qualifikation als Kunstwerk, als kontextabhängig, nämlich insbesondere als von der Gesellschaft bestimmt und nicht als «reale Eigenschaft der Dinge». ${ }^{62}$ Die ästhetische Funktion ist für ihn nur ein «Faktor der Lebenspraxis», der fließend in andere Funktionen übergehen kann und «einen größeren Wirkungsbereich als die Kunst selbst» besitzt. ${ }^{63}$

Auf Grund der gesellschaftlichen Bestimmung des Ästhetischen, der aktuellen ästhetischen Norm, können Kunstwerke im Laufe ihrer Geschichte ihre ästhetische Funktion ändern, sie gewinnen oder verlieren. ${ }^{64}$ Die Forschung kann daher ursprüngliche ästhetische Funktionen von Kunstwerken wieder entdecken und zwar im Kontext des jeweiligen Verständnisses von Kunst. ${ }^{65}$ Ein Funktionswandel der Kunstwerke betrifft auch immer die jeweilige Normvorstellung von Kunst, die in ei-

${ }^{60}$ BAsChET 1996, 20: spricht «image-objet», weil es zugleich darstellendes Kunstwerk und benutzbarer Gegenstand sei.

${ }^{61}$ Cf. Busch 1997A.

${ }^{62}$ MukarovsKŕ 1967, 13: «Aber die aktive Qualifikation zur ästhetischen Funktion ist keine reale Eigenschaft des Gegenstandes, selbst wenn er absichtlich auf die ästhetische Funktion hinzielt, sondern sie tritt nur unter bestimmten Umständen, nämlich in einem bestimmten gesellschaftlichen Kontext zutage.»Cf. ib., 18: Kunst definiert durch dominante ästhetische Funktion. Cf. ib., 29: «1. Das Ästhetische ist weder eine reale Eigenschaft der Dinge noch eindeutig an bestimmte Eigenschaften der Dinge gebunden. 2. Die ästhetische Funktion von Dingen ist nicht völlig in der Macht des Individuums, wenn auch aus rein subjektiver Sicht etwas ohne Rücksicht auf seine Gestaltung eine ästhetische Funktion erhalten (oder verlieren) kann. 3. Die Stabilisierung der ästhetischen Funktion ist die Sache eines Kollektivs, und die ästhetische Funktion ist Bestandteil des Verhältnisses zwischen dem menschlichen Kollektiv und der Welt.» Cf. Schamschula 1979, 245-255: zu Jan Mukarovskýs Funktionsbegriff. Cf. Schamschula 1998. Cf. LAUer 1979: zu Jurij Nikolaevic Tynjanovs literaturtheoretischem Funktionsbegriff. Cf. VeldHUES 1998. Cf. Olechnowitz 1981, S. 220-293: zum Autonomiebegriff Mukarovský und Tynjanov.

63 MuKarovsKÝ 1967, 12: «Darüber hinaus hat die ästhetische Funktion einen weit größeren Wirkungsbereich als die Kunst selbst.» Ib., 14-15: Die ästhetische Funktion sei kontextabhängig, daher fließend in andere Funktionen übergehend, dialektisch. Ib., 112: «Das Ästhetische [...] ist ein bedeutsamer und vielseitiger Faktor der Lebenspraxis.»

${ }^{64}$ Cf. MuKarovsKÝ 1967, 13: Kunstwerke als privilegierte Träger würden ihre ästhetische Funktion verlieren oder wiedergewinnen können. Cf. Busch 1987, 7: «Die ästhetische Funktion ist durch die ästhetische Norm bestimmt. Normenwandel erzeugt Funktionswandel.»

${ }^{65}$ MuKarovsKÝ 1967, 14: Forschung könne ursprüngliche ästhetische Funktionen wieder entdecken. Ib. 122: «Sie [die Funktionen allgemein] dürfen nicht einseitig auf das Objekt projiziert werden, sondern es muss vor allem das Subjekt als ihre lebendige Quelle Berücksichtigung finden.» 
nem reziproken Verhältnis stehen. ${ }^{66}$ Die Funktionsgeschichte trägt dazu bei, einerseits den Kunstbegriff zu historisieren und zum Forschungsgegenstand zu machen, andererseits die Definition von «Kunst-Geschichte> vom Kunstbegriff abzukoppeln und das Fach auf eine allgemeinere Bild- oder Objektgeschichte auszurichten. ${ }^{67}$

Plädierte man in der Kunstwissenschaft für einen rein ästhetischen, negativen Funktionsbegriff, nämlich für das ‘Zweckfreiheit` genannte Unding, würden etwa die Gebrauchskunst der Propaganda und Werbung, das so genannte 〈Kunsthandwerk〉 oder gar die meisten Formen zeitgenössischer Ausstellungskunst aus dem Rahmen einer derart normierten Bildgeschichte fallen. ${ }^{68}$ Die Idee künstlerischer Autonomie, jenes Amalgam historisch divergenter Begriffe und Vorstellungen wie etwa 〈licenza〉, 〈Freiheit〉, ২Zweckmäßigkeit ohne Zweck〉 oder 〈Idealität», das sich im 18. Jahrhundert herausbildet, wirkt sich historisch exklusiv aus, so dass oft zu lesen ist, 〈Kunst $>$ fange erst in der frühen Neuzeit an. ${ }^{69}$ Bereits die Definition des Gegenstandsfeldes der Kunstwissenschaft durch den modernen Begriff 〈Kunst s schließt Produkte nicht westlicher, vormoderner und 〈primitiver> Kulturen a priori aus: Es ist nicht der Fall, dass sie keine 〈Kunst besäßen, sondern dass sie keine 〈Kunstwissenschaft $〉$ oder 〈Kunstphilosophie〉 institutionalisieren, die ihre Kulturerzeugnisse als «Kunst $〉$ bestimmen. Weil der kategorische Oberbegriff 〈Kunst〉 die unterschiedlichsten und widersprüchlichsten Gegenstände und Vorstellungen benennt, kann erst gar nicht von der Funktionalität oder Zweckfreiheit der Kunst im allgemeinen gesprochen werden. ${ }^{70}$ Wenn man den Kunstbegriff auf 〈Autonomie〉 und ¿Zwecklosigkeit〉 reduzieren wollte, würde man kein positives und produktives Kriterium für Kunst schaffen, denn bloße Nutzlosigkeit kann das Kunstwerk nicht ausmachen und kann einem menschlichen Produkt kaum zugeschrieben werden, und dem Funktionssystem - sozialgeschichtlich: der bürgerlichen Zweckrationalität - würde man ebenso wenig entrinnen. ${ }^{71}$ Oder wie Jan Mukarovský beobachtet: Die so genannte 〈reine Kunst〉 ist so monofunktional und monokausal wie eine Maschine, deren Charakter sie ja

\footnotetext{
${ }^{66}$ BusCH 1987, 5: «Historisch ändert sich die ästhetische Funktion durch einen Normenwandel in der Gesellschaft; sie verfügt die Funktionsanforderungen an das Kunstwerk.» Ib.: «Wandel in den Funktionsanforderungen ist einer der Haupttriebskräfte für die Kunstentwicklung.»

${ }^{67}$ Cf. BöHME ET AL. 2000, 73: «In der Kulturwissenschaft [Aby M. Warburgs] gibt es keine reine, sondern nur materiale Theorie. [...] Es darf kein dogmatisches Anwendungsverhältnis von Theorie auf die Gegenstandsebene geben. Jede Theorie muss aus dem Material erarbeitet werden. Das ist eine für die Theorie der Kulturwissenschaft gültige Einsicht.»

${ }^{68}$ Cf. BÄTSCHMANN 1997.

${ }^{69}$ Cf. Olechnowitz 1981.

${ }^{70}$ Cf. SCHMÜCKER 2001, 18
}

gerade ablehnt. ${ }^{72}$ Nicht zuletzt würde die Vorstellung von Zweckfreiheit davon ausgehen, dass Gegenstände Funktionen haben können oder nicht, als ob es sich um physische Qualitäten handelte, was auszuschließen ist.

Eine positiv verstandene «ästhetische Funktion〉 bedürfte jedenfalls weiterer Differenzierungen: Denn manieristischen Kunststücken, impressionistischen Salongemälden und zeitgenössischer Galeriekunst ist nur oberflächlich gesehen ein ähnlicher Anspruch des L'art pour l'art gemein, da die jeweiligen Bedingungen und Ausdrucksweisen künstlerischer Selbstbestimmung voneinander differieren. «Freiheit〉, 〈Selbstbestimmung〉, 〈Unabhängigkeit), 〈Autonomie> sind, historisch gesehen, negative Begriffe oder gar bloße, wenn auch wirksame Parolen, die dem Befreiungsakt der Kunst und Künstlerschaft aus sozialen, ökonomischen, politischen, religiösen Zwängen dienen. ${ }^{73}$ Die bloße, so genannte 〈künstlerische Autonomie`, die fragwürdige Kategorien voraussetzt wie 〈Innen〉 und 〈Außen〉 und in eine gedankliche Sackgasse führt, muss ideologiekritisch und sozialgeschichtlich als eine Funktion unter anderen betrachtet, mit weiteren Funktionen verknüpft und auf explizite und implizite Ziele, beispielsweise ökonomischer, sozialer oder politischer Art, zurückgeführt werden. ${ }^{74}$

\section{Objektivität}

Die Funktionsgeschichte scheint jedoch, trotz begrifflicher Unschärfe, zu funktionieren. Vor der Perspektive sich selbst genügender oder machtpolitisch motivierter Methodendiskussionen und im Bewusstsein, dass Kunstforschende keine Atomreaktoren entwerfen, mag es berechtigt scheinen, die praktische Relevanz des Strebens nach naturwissenschaftlich geprägter Gewissheit und Genauigkeit zu bezweifeln. Pragmatischer ist es, einer etablierten Vorgehensweise wie der funktionsgeschichtlichen Analyse von Kunstwerken - etwa nach der populären informationstechnischen Devise never change a running system - zu folgen, so lange sie konkrete Erkenntnisgewinne einspielt, wie dies beispielsweise im Studium von

\footnotetext{
${ }^{71}$ LuHMANN 1998, 247: «Nutzlosigkeit ist nur die andere Seite der Form des Nutzens». Cf. ib., 245: Schönheit als «zweckloser Selbstzweck». Cf. Busch 1997C, 799: «Zweck und Zweckfreiheit der Kunst zwei Seiten einer Medaille».

${ }_{72}$ MuKarovsKú 1986, 83

${ }^{73}$ Cf. SCHMÜCKER 2001, 20: «Die Rede von der Autonomie der Kunst ist lediglich eine façon de parler: eine gezielte terminologische Übertreibung, die die relative Unabhängigkeit der Künstler von tradierten Normen und von den Vorgaben von Auftraggebern hervorzuheben vermag. [...] Als kunstphilosophischer These jedoch gebührt ihr ein Platz im Kuriositätenkabinett der Ästhetikgeschichte.»

${ }^{74}$ Cf. WolfzetTel / Einfalt 2000-2001: zur künstlerischen Autonomie.
} 
Wandmalereien in Fürstenresidenzen oder von liturgischem Kunsthandwerk eindeutig der Fall ist. ${ }^{75}$ Gerade eine nicht streng definierte, methodisch induktive Form der Funktionsgeschichte kann sich durch diskursives trial and error weiter verfeinern und sich Einzelbedürfnissen anpassen.

Den sozusagen methodisch «weichen oder «schwachen funktionsgeschichtlichen Forschungsansatz zeichnet der Vorteil aus, dass er sich, wenn etwa das Quellenmaterial als spärlich oder überwältigend herausstellt, auf den Commonsense und die alltägliche Erfahrung der Objektnutzung des Forschenden und Lesenden berufen kann. ${ }^{76}$ Die Vorstellungskraft, die in der historischen Forschung und Wissensvermittlung eine grundlegende Rolle spielt, gibt ein zwar nur ungefähres, doch ein relativ brauchbares Bild davon, wie etwa die Enfilade eines Palastes durchschritten oder das Reliquiar in einer Prozession getragen werden mochte oder welche Aufgaben ein Thron zu erfüllen hatte. Eben solche allgemein verständlichen, mentalitätsgeschichtlich rekonstruierbaren, von einer Vorzeit in die Gegenwart übersetzbare Handlungsmuster stiften Bedeutung und unterstützen das Verständnis.

Sollte es in der Kunstgeschichte als historischer Bildwissenschaft oder Kulturgeschichte um eine möglichst facettenreiche, mikrohistorisch fundierte Rekonstruktion der Erscheinung und Bedeutung der Bildwerke in ihrem Lebenszusammenhang, um eine Art antimuseale 〈Vergegenwärtigung〉 gehen, so könnte die Funktionsanalyse und Kontextforschung die Augen für mannigfaltige, bis dahin verschollene Sinn- und Nutzungsbezüge öffnen. ${ }^{77}$ Es handelt sich dabei, im Sinne der Hermeneutik, um eine interessengeleitete und -formende, nicht sozusagen <objektive> Rekonstruktion historischer Zusammenhänge, die aber, wie in der Kriminalistik, eine Annäherung an einen Sachverhalt für möglich hält und anstrebt, ohne ihn in allen seinen Aspekten erklären zu können. ${ }^{78}$ Gälte es, die analysierte anthropo-

${ }^{75}$ Cf. Busch 1997C: zu den Anwendungsgebieten der Funktionsgeschichte. Cf. Didi-HubERMAN 1996, 75: zum pragmatischen Funktionsbegriff in der heutigen Kontextforschung.

${ }^{76}$ Cf. Gombrich 1993, 116: «Ich will keine [Methode]. Ich will lediglich gesunden Menschenverstand. Das ist meine Methode.» GEERTZ 1987C.

77 Sedlmayr 1958, 7: «[Die Einsicht ist wesentlich, dass die] historische Aufgabe der Kunstgeschichte die Vergegenwärtigung der Kunstwerke und dass das Kunstwerk eine Welt im Kleinen ist. Diese Vergegenwärtigung geschieht im Prozess der Interpretation, und diese Interpretation von Kunstwerken ist keineswegs nur gedankliche Deutung, sondern eine Umwandlung der Anschauungen, primär im Interpreten, sekundär im Betrachter.»Cf. FoRssmAN 1974, 52: «Erzeugt wurde das unhistorische Gefühl der ständigen Gegenwart der Kunstwerke durch die museale Bewegung des 19. Jahrhunderts, als man die Kunstwerke aus ihren geschichtlichen Zusammenhängen löste und dem Publikum als zeitlos und ewig gegenwärtig präsentierte.» ZiJLMANS / HALBERTSMA 1995, 26-27: «Möglicherweise liegt hier die tiefste Wurzel kunsthistorischer Forschung: dem Kunstwerk seinen authentischen Kontext zurückzugeben. Dieser Kontext ist jedoch unwiderruflich verloren; seine Rekonstruktion ist lediglich im kunsthistorischen Text möglich.» logische Bedeutung eines historischen Kunstgegenstandes zu vermitteln, dürften kontextuelle, aktualisierende oder performative Mediengattungen, beispielsweise der Historienfilm oder das Erlebnismuseum, sich als effektiver erweisen denn der wissenschaftliche Text. Je mehr die anthropologische Rekonstruktion sich im Detail der historischen Realität nähert, umso mehr gleicht sie unserer trivialen Wirklichkeit, was keine verzweifelte Suche nach spannenden, dramatischen, extraordinären, erstaunlichen, originellen Ereignissen, Akteuren, Zeugen, Interpretationen einer marktorientiert und massenmedial betriebenen Kunstgeschichte wettmachen könnte; oder wie in Paul Veynes positiver Formulierung: «Die Banalität der Vergangenheit besteht aus unbedeutenden Einzelheiten, die durch Vermehrung schließlich dennoch ein sehr unerwartetes Bild ergeben.» ${ }^{79}$

Als ästhetisches Kulturprodukt kann das Kunstwerk zu einem gratifizierenden Gegenstand der «dichten Beschreibung> werden, wie Clifford Geertz in der Ethnologie das interpretierende Verstehen nennt, das dem Systemdenken die experimentelle Generalisierung von Einzelfällen vorzieht, wie auch Gegenstand einer bewusst subjektiven und doch objektnahen steilnehmenden Beobachtung> im Sinne Bronislaw Malinowskis (1884-1942) werden, die mittels einer fundierten Narration beim Leser eine entsprechende Teilnahme zu erwirken sucht. ${ }^{80}$ Wie Geschichtsbücher und -ausstellungen belegen, spielen Kunst und Kunstgeschichte eine wesentliche Rolle in dem Wunsch, sich eine Vorstellung von der Vergangenheit, ein poetisches Bild der Geschichte zu machen, was im Falle der kunsthistorischen Deutung zu einem Bild eines Bildes wird.

Kunstwerke und Bilder wären dann weder bevorzugte noch gering geschätzte Gegenstände der Forschung, die mittels harter Fakten erklärt werden könnten, sondern wären nicht weniger facta, das heißt hergestellte Konstrukte und Interpretationen, als jeder andere, nicht ästhetische Sachverhalt. ${ }^{81}$ In diesem Sinne gibt es keinen wesentlichen Unterschied zwischen Kunst und Kontext, denn jeder Text ist des anderen Kontext, wie etwa der new historicism bekräftigt. ${ }^{82}$ Wenn Kunstwerk und Kontext in ihrer Vieldeutigkeit gleichgesetzt werden, kann dem Kontext keine prädominierende faktische Aussagekraft zugesprochen werden, auf welche eine eindeutige Interpretation des Kunstwerks sich stützen könnte. ${ }^{83}$ Daher können 〈Kunst〉 be-

78 Cf. Daniel 2001, 285-296: zu Carlo Ginzburg und der Mikrohistorie. Cf. Bätschmann 1994. Cf. BocK ET AL. 2000: Funktionsgeschichte sei keine Totallösung.

${ }^{79}$ VEYNE 1996, 18: «la banalité du passé est faite de particularités insignifiantes qui, en se multipliant, n'en finissent pas moins par composer un tableau très inattendu.»

${ }^{80}$ GeERTz 1987A, 21: «Sie [die Kultur] ist ein Kontext, ein Rahmen, in dem sie verständlich - nämlich dicht - beschreibbar ist.» Cf. GEERTZ 1987в. Cf. DANIEL 2001, 233-254: zur Ethnologie.

${ }^{81}$ Cf. Forssman 1974, 53 
ziehungsweise 〈Kunsttheorie〉 und 〈Kunstwerk〉 nicht a priori in ein hierarchisches und kausales Verhältnis gesetzt werden, sondern es ist angebrachter, nach der jeweiligen Funktion von Einzelphänomenen zu fragen, seien sind geschrieben, gebaut, gemalt und dergleichen. Beispielsweise wären, aus diesem Blickwinkel gesehen, Bilder nicht als Abbilder gesellschaftlicher Kontexte zu verstehen, sondern als ein aktiver Bestandteil des Kontextes, so dass gesellschaftliche Verhältnisse auch umgekehrt als Ausdruck und Wirkung von Kunst angesehen würden. ${ }^{84}$ Wie Norman Bryson beobachtet, stehen der visuelle Text des Kunstwerks und der Kontext, die beide vielmehr hergestellt als gegeben sind, nicht nur in einer Scheinopposition zueinander, sondern die Einzelbildanalyse selektiert oftmals die relevanten kausalen Kontextfaktoren zu ihren Gunsten so vor, dass es in der kunsthistorischen Kontextforschung zu einer Umkehrung von Ursache und Wirkung, zu einer Tautologie oder einer sich selbst erfüllenden Voraussage kommen kann, die schließlich zum Sinnbild, zum Pars pro Toto oder zur Illustration großer Kontexte, wie etwa der Epochen oder Stile, erhoben wird. ${ }^{85}$

Aus einer bewusst interpretierenden und in ihrer Detailfokussierung immer ganzheitlichen Geschichtsschreibung resultiert, dass es keine «nackten` Tatsachen und Gegenstände gibt, sondern nur Knoten in einem durch Interpretation rekonstruierten Zeichennetz, und dass keine Unterscheidung der Geschichtsfächer sinnvoll ist, da Gegenstände - ganz wie in unserer gegenwärtigen Erfahrung - nicht monokausal, sondern immer «interdisziplinär entstehen und vergehen. Daher sollte jede Disziplin, jeder Ansatz, jede Methode in der Bildwissenschaft willkommen sein, die zum Verständnis des Gegenstandes beiträgt. Dementsprechend löst die Kunstgeschichte ihre methodischen Grenzen auf, um in die Kul-

${ }^{82}$ Cf. Zijlmans / Halbertsma 1995, 27: «Der prinzipielle Unterschied zwischen Kontext und darin befindlichem Gegenstand ist weniger groß, als man denkt, denn der Kontext ist nicht von vorneherein vorhanden, sondern wird - wie das Kunstwerk - vom Interpreten bei der Interpretation produziert. Der Kontext ist also genauso interpretationsbedürftig wie das Kunstwerk selbst.» Cf. Kemp 1991, 98: «So wie der Text immer im Kontext situiert ist, so befindet sich der Kontext auch immer im Text wieder.» Cf. BöHME ET AL. 2000, 14-16: zum new historicism und zu Stephen Greenblatt.

${ }^{83}$ Cf. BätschmanN 1992, 48-50.

${ }^{84}$ Cf. BRYson 1994, 72: «A harder social analysis might treat the pictures incidentally, in passing, as one sort of evidence among many - if one is going to do social history, why privilege works of art in such a way that the findings of historiography must be bound to the mise-en-scène of painting?» Cf. z. B. BASCHET 1996, 23: Bilder seien nicht ein blosses Abbild der Gesellschaft. Cf. BeLting 1986.

${ }^{85}$ BRYsON 1994, 66-67: Um der Scheinopposition zwischen visuellem Text und Kontext zu überwinden, sollte man eher von «frames», bewussten «Einrahmungen〉 oder Kontexturalisierungen sprechen. Ib., 72: «synecdoche: context is necessarily conceived as a totality; it is everything in the world that could possibly count as context - everything, that is, except the visual text.» Ib., 73: «picture and commentary stand in a mimetic rather than an anatic relation, the context copying the visual text, and the visual text iconizing or imaging the context.» turgeschichte einzugehen, die selbst schon eine «kunstgeschichtliche〉 Perspektive auf ihre historischen Gegenstände einnimmt, wenn sie sie als ein illustratives, symbolisches oder allegorisches Pars pro Toto größerer Zusammenhänge einsetzt. ${ }^{86}$ Da 〈Kunst〉 als ein spezifischer historischer Kontext zu verstehen ist, wäre zu überlegen, ob die Kunstgeschichte in eine allgemeine «Objektgeschichte> überführt werden müsste, um sie von vorgefassten begrifflichen Verengungen, wie etwa 〈Kunst〉 oder 〈Bild〉 im Falle von Kunst- und Bildwissenschaft, die beide große kulturelle Gegenstandsfelder ausschließen, grundsätzlich zu befreien.

Wie der Gegenstand erklärt, verstanden werden und überhaupt erscheinen soll, hängt von der (damaligen wie heutigen) Perspektive, dem Interesse, der Fragestellung, dem Zweck der Übung, den Grenzen und Möglichkeiten eines kollektiven «Denkstils» ab. ${ }^{87}$ Es wäre aber ein Missverständnis, wollte man dem forschenden Subjekt alle Macht über den historischen Gegenstand zusprechen, denn dieser zeigt, wie die Erfahrung lehrt, Präferenzen und eigene Gesetzmäßigkeiten, Widerstand und Eigenwilligkeit gegenüber dem Interesse der Forschenden. ${ }^{88}$ Jeder Gegenstand erfordert mehr oder weniger eigene Ansätze und regt $\mathrm{zu}$ je eigenen Fragen an. ${ }^{89}$ Aus diesem Grund sind Methoden in der Praxis nicht beliebig anwendbar, sondern auch objektabhängig. Historisches Objekt und forschendes Subjekt bestimmen sich also gegenseitig. ${ }^{90}$ Vielleicht ließe sich die Schnittmenge und Reibungsfläche, wo der Gegenstand sich dem Subjekt widersetzt und dessen theoretische Willkür einschränkt, 〈Objektivität` nennen.

\section{Form}

Der kunsthistorische Funktionsbegriff wird trotz des angedeuteten Definitionsbedürfnisses offen und flexibel bleiben müssen. Da Kunstobjekte und ihre Nutzungsweisen einem historischen Wandel unterworfen sind, wird es sich in der Kunstwissenschaft, laut Werner Busch, nicht um einen «mechanistischen» und unidirektionalen, sondern um einen «dynamischen Funktions-

${ }^{86}$ Cf. Preziosi 1989, 34: «Currently, the discipline of art history seems like a kind of department store of methodological options from which we might choose or mix and match, according to the dictates of personal taste, selecting particular instruments suited to interlock with the configurations of perceived problems.»

${ }^{87}$ Cf. FLECK 1980: zum «Denkkollektiv» und zum «Denkstil». Cf. DANIEL 2001, 17: Die Objekte der Kulturgeschichtsschreibung seien abhängig von der Sichtweise sowohl der damaligen Zeitgenossen wie auch des heutigen forschenden Subjekts.

${ }^{88}$ Cf. GeERTZ 1987A, 260: «Gesellschaften bergen wie Menschenleben ihre eigene Interpretation in sich; man muss nur lernen, den Zugang zu ihnen zu gewinnen.»

${ }^{89}$ Cf. Gombrich 1993, 116: «Jede Frage bedarf einer anderen Methode, damit man zu einer Antwort kommt.»

${ }^{90}$ Cf. DANIEL 2001, 395-396: zur Wechselwirkung zwischen Subjekt und Objekt in der Kulturgeschichte. 
begriff» handeln müssen. ${ }^{91}$ Die Frage nach den Nutzungsweisen und -möglichkeiten lässt das Kunstwerk in einem Lebenszusammenhang erscheinen, welcher sowohl die Form wie auch die Bedeutung des Objekts bestimmt und umgekehrt. Die Kunstwerke, denen Ernst $\mathrm{H}$. Gombrich einen ökologischen Lebensraum zuspricht, wirken wie Tiere in ihrem Biotop auf ihren Lebenszusammenhang und Kontext, der sie bestimmt, zurück. ${ }^{92}$

Diese reziproke Abhängigkeit findet sich zwischen den Begriffen 〈Form〉 und 〈Funktion〉. Sie kommt zu Tage, wenn man beispielsweise fragt, was früher kam: der Bilderkult oder das Kultbild? das Zeremoniell oder der Palast? ${ }^{93}$ So wie etwa Intention und Rezeption, lassen sich Zweck und Gebrauch eines Kunstwerks nicht zwingend und ausschließlich definieren, denn, um das bekannte Credo des amerikanischen Architekten Louis HenriSullivan (1856-1924) von 1894 an- und umzuwenden: Form bedingt Funktion, und Funktion bedingt Form. $\cdot{ }^{94}$ Form und Funktion sind miteinander verquickt: Das Altarbild erhält eine funktional bestimmte Form, die umgekehrt Altarbilder als funktionale Gattung erst kenntlich und brauchbar macht. Die Funktion formt den Gegenstand, dessen Gestalt als ein zu interpretierendes Zeichen für die selbe, aber auch für eine andere Funktion angesehen werden kann, und so weiter. In historischer Rückschau sind Ursache und Wirkung oft kaum voneinander zu unterscheiden, wie etwa im Falle des wechselseitigen Einflusses zwischen Architektur und Liturgie.

Dem Zweifel Horst W. Jansons, dass ein rein funktionales oder ein rein unfunktionales Objekt oder

${ }^{91}$ Busch 1987, 6: «Wie verwenden keinen statischen, mechanistischen Funktionsbegriff, sondern einen dynamischen. [...] Der dynamische Funktionsbegriff geht davon aus, dass sich die Funktionen des Kunstwerkes historisch wandeln, dass damit aber auch das Kunstwerk selbst in seiner Bestimmung historischem Wandel unterliegt.» Cf. Busch 1997в, 19. Cf. Didi-Huberman 1996, 84-86: Der Funktionsbegriff sei «dialektisch», uneinheitlich (psychoanalytisch), unidealisiert (formalistisch) und nicht teleologisch (anthropologisch).

${ }_{92}$ Gombrich 1993, 62-63. Cf. Prange 1998 und Kemp 1991.

${ }^{93}$ Cf. z. B. Kimpel / SucKale 1997A, 43: «Die Liturgie bestimmte die Form der Kathedrale, und umgekehrt bestimmte die Kathedrale die Form der Liturgie.» Cf. Kerscher 1990, 98: Dies sei eine unbeantwortete Frage; «hat das Zeremoniell am päpstlichen Hof den Bau des Palastes beeinflusst, oder bestand die Architektur zuerst und das Papstzeremoniell entwickelte sich in ihr?». Ib., 110: «Man könnte auch fragen, was früher existiert hätte, das Zeremoniell oder der Papstpalast, der den Raum für das Zeremoniale bot».

${ }^{94}$ Cf. Janson 1982, 5. Cf. Belting 1995, 83: «[Eine] Funktion kann eine vorhandene Bildform verwandeln, und diese kann ihrerseits wieder neue Funktionen übernehmen, und so weiter. Es ist überhaupt nur sinnvoll, nach Funktionen zu fragen, wenn das Erkenntnisse für die visuelle Struktur eines Bildes einbringt. Wenn sie weder die Existenz noch die Form eines Bildes erklärt, muss «Funktion` auch nicht als eigener Begriff eingeführt werden, sondern genügt als zusätzliche Information.» Cf. BAsCHET 1996, 17: zu intendierten und nicht vorgesehenen Objektnutzungen.
Kunstwerk existieren könne, fügt Stanislaus von Moos die Bemerkung hinzu, dass es keine einfache Kausalverbindung zwischen Form und Funktion gebe: Ein reiner architektonischer Funktionalismus, pure Zweckbauten etwa, seien eine Illusion, weil jede Formfindung von vielen und nicht zuletzt von ästhetischen Faktoren abhinge; umgekehrt könne eine funktionalistische Ästhetik, etwa im Falle rein ornamentaler Wehrhaftigkeit einer Burg, Zweckmäßigkeit suggerieren und diese gar so erfolgreich vortäuschen, dass sie den Feind abschreckt und damit ihren scheinbaren Zweck realisiert. ${ }^{95}$ Dass Funktionalität auch ein bloßes, gar post festum interpretiertes Zeichen, und dessen Träger im Gegenteil unfunktional sein können, ist beispielsweise an der architecture parlante eines Claude Nicolas Ledoux (17361806) oder an der machine à habiter eines Le Corbusier (1887-1965) abzulesen. ${ }^{96}$

\section{Kategorien?}

Der kunstwissenschaftliche Funktionsbegriff lässt sich weiter differenzieren. Vier Aspekte charakterisieren, nach Werner Busch, das Kunstwerk: Seine Funktionen seien komplex, mehrdeutig (polysemantisch), wandelbar und könnten gleichzeitig am selben Objekt auftreten (kompatibel). Des Weiteren unterscheidet er vier Klassen von Primärfunktionen: die religiöse, die ästhetische, die politische und die abbildende Funktion. ${ }^{97}$ Dieser Katalog könnte aber erweitert oder verändert werden, je nachdem welche Benutzung zu einer primä-

${ }^{95}$ Moos 1974, 213-214. Ib., 211-213: Eindruck oder Ästhetik der Funktionalität gehe über die mechanische Zweckmäßigkeit hinaus. Ib., 214: «Das Wissen um die Funktion allein führt nicht geradewegs zur einzig richtigen Form; diese ist vielmehr zu einem großen Teil durch Faktoren determiniert, die jenseits einer rein utilitären Logik liegen. Fast immer liegt ihr eine ästhetische Entscheidung zugrunde.» Cf. ib., 207-215: zum Zweckbau.

${ }^{96}$ Cf. Posener 1977, 20: «Rationalisierung post festum ist eine Methode des Funtkionalismus»; Ib., 20-21: «Der Funktionalimus war eine künstlerische Bewegung, eine stilschaffende Bewegung, welche unter bestimmten historischen entstanden war. Er war nicht zweckrational, aber seine Theorien waren auf die Zwecke bezogen. Er verfuhr nicht analytisch, aber seine Methode war Analysis, vielmehr: sie wollte es sein. Seine Theorie bereits versuchte, den Zweck in den Dienst der Form zu stellen, obwohl sie es oft umgekehrt ausdrückte. Sie versuchte, eine zwingende Beziehung zwischen Zweck und Form herzustellen, indem sie von mechanischen Vorgängen ausging. Sie bedachte nicht, das Wohnen, zum Beispiel, kein mechanischer Vorgang ist. Das machte die Theorie für die Praxis unbrauchbar und liess dem Architekten keine Wahl: Er musste jedesmal den Kurzschluss zur Kunst machen.» Cf. VogT 1977, 25-26, cap. Der Dampfer als ‘Zeichen〉 der modernen Architektur.

${ }^{97}$ Busch 1987, 6. Busch 1997в, 16-17: zu den Merkmalen und Kategorien der Funktionen von Kunstwerken. Cf. De ZuRKo 1957в, 7: «The idea of function is not a simple one. Function may be objective or subjective.» 
ren Funktion erhoben wird: $\mathrm{Zu}$ den hier vier implizierten kulturellen Tätigkeitsbereichen des Menschen - Religion, Kunst, Politik und Wissenschaft - ließen sich weitere grundlegende Motivationen für die Herstellung von Kunstwerken und Bildern hinzufügen, wie etwa evolutionsbiologische oder ökonomische Funktionen. ${ }^{98}$ Die Funktionsklassen mag man ebenfalls nach anderen Prinzipien auffächern wollen, etwa nach biologischen und kulturellen Bedürfnissen, die Bilder zu befriedigen haben, nach Institutionen und Interessengruppen, die Kunst produzieren und konsumieren, oder nach sozialen Normhandlungen, mit welchen sich die Funktionen von Kunst beschreiben lassen - es kommt darauf an, was zum Unterbau erklärt wird. Dieser bleibt jedoch wackelig, weil dessen dekontextualisierte Oberbegriffe wie 〈Kult〉, 〈Erinnerung〉, 〈Schmuck〉 selbst definitionsbedürftig sind.

Von festen Funktionskategorien ist daher abzusehen. Wie Edward Robert De Zurko bemerkt, impliziert der Funktionalismus ein pluralistisches und nicht monistisches Wertesystem: So wird beispielsweise die Funktionalität von Architektur, je nach historischer Situation, ethisch, sozial oder metaphysisch beurteilt. ${ }^{99}$ Funktionen erscheinen zwar sowohl als gattungsbildend wie auch als gattungsabhängig: Eine formale Bild- oder Objektgattung kann einerseits funktional bestimmt, die Funktion andererseits aus der formalen Gattung abgeleitet werden. ${ }^{100}$ Doch sind die sozialen Funktions- und Bedeutungssphären, in denen ein Objekt steht, nicht fest definiert, sondern wandelbar. Hans Belting spricht daher nicht von starren oder gar mechanistischen «Funktionsformen», sondern von funktional definierten «Existenzformen» als «Existenzbedingungen» der Bilder oder Kunstgegenstände, die wie andere sozialhistorische Phänomene aus einer Mischung von Regel und Veränderung bestehen, weswegen er unter «Funktion» «den Umgang mit Bildern und den Bedarf

\footnotetext{
${ }^{98}$ Busch 1987, 6: zu den unterhaltenden, erzieherischen, bildenden, therapeutischen, erotischen Funktionen. Cf. z. B. BAscheT 1996, 21: zu den politischen, sozialen, rechtlichen und religiösen Funktionen. Cf. ib., 17-21: zu Norm, Intention, Benutzungsweise und Rolle als spezifische Kategorien der Funktionsanalyse mittelalterlicher Kunst. Cf. SCHMÜ CKER 2001, 28, fig. 1, Schema der Funktionen der Kunst.

${ }_{99}^{9}$ De Zurкo 1957в, 232: «Functionalism implies a pluralistic, not a monistic, system of values. Functionalist criticism, observed in historical dimension, manifests a tendency to evaluate historical architecture largely in terms of moral, ethical, social, and often metaphysical ultimate values, whereas in evaluating contemporary architecture there is a tendency for the critic to stress primary or immediate values such as economy, ease of circulation, sanitary features, ease of maintenance, or good light and ventilation.»

${ }^{100}$ Cf. z. B. Belting 1995, 98: «Deshalb ist das Problem der Unterscheidung von verschiedenen Bildfunktionen und entsprechend verschiedenen Funktionsbildern ein Problem der Priorität, wo eine Bilderfindung authentisch verwendet und wo sie in andere Aufgaben übertragen wurde. [...] Jede starre Klassifikation würde die Komplexität dieser Zusammenhänge verfehlen. Konventionen pflegen sich ohnehin auszubreiten, ohne sich an Funktionsschranken zu halten.»
}

nach solchen, also Konventionen aus der Sozialgeschichte der Kunst, beziehungsweise historische Bedingungen» versteht. ${ }^{101}$

Fraglich ist daher auch, ob es ein System von Funktionen geben kann. Der Organizismus, der dem soziologischen und ästhetischen Funktionsbegriff zu Grunde liegt, fördert die Idee, dass das gesellschaftliche Ganze, welches die Kunstfunktionen bestimmt, ein geschlossenes und festes System von Institutionen sei, wohingegen eine Gruppe variabler Faktoren zutreffender erscheint, da die Produktion und Konsumption von Kunst institutionell, juristisch und ökonomisch weniger stark geregelt ist als andere gesellschaftliche Tätigkeitsfelder. ${ }^{102}$ Wie Georges Didi-Huberman betont, ist die soziologische Vorstellung eines einheitlichen Gesellschaftsganzen, dem ein Bedürfnissystem entspräche, das unter anderem durch Bilder befriedigt und zugleich dargestellt werde, von Kritikern als ein «epistemologischer Mythos» bezeichnet worden..$^{103}$

Schließlich sind nicht alle Funktionen allen Künsten gemeinsam, da beispielsweise die abbildende Funktion nicht zu den primären Zwecken der Architektur zu zählen ist. ${ }^{104}$ Eine flexible Differenzierung der Funktionstypen ist daher notwendig, damit das Kunstwerk in seiner multifunktionalen Form und seinen vielseitigen Bedeutungen kontextuell, das heißt tendenziell präziser und facettenreicher, fassbar wird. Auf Grund seiner Relativität kommt dem Funktionsbegriff jedenfalls eine großes Anwendungspotential zu, wie es Niklas Luhmann (1927-1998) in Hinblick auf die Soziologie formuliert hat, demzufolge «die Erweiterung des Fassungs-

${ }^{101}$ BeLtiNg 1995, 25: «Wir können daher von Existenzformen des Bildes sprechen, die zugleich dessen Existenzbedingungen waren. Das gerahmte Bildobjekt bedurfte einer Funktion, um existieren zu können beziehungsweise akzeptiert zu werden. Existenzformen des Bildes sind folglich solche, die auf eine reale oder symbolische (soziale) Funktion bezogen sind und sich in der spezifischen Vermittlung eines Bildinhalts ausdrücken. Man könnte auch von Funktionsformen sprechen, doch würde ein solcher Begriff allzu sehr die Vorstellung von äußerlicher Zweckbindung im engere Sinne determinieren, die nur einen Sonderfall darstellt. Wir verstehen daher unter «Funktion` den Umgang mit Bildern und den Bedarf nach solchen, also Konventionen aus der Sozialgeschichte der Kunst, beziehungsweise historische Bedingungen.»

${ }^{102}$ Cf. De ZuRKo 1957в, 4: «The term <organic is related to <functionalism >» Ib., 20: aristotelischer Organizismus. Cf. BusCH 1997в, 1819 und Jones 1996. Cf. BAsChet 1996, 15 und Thiel 1973, 513-514. Cf. BELL 1997, 27-29: zu den funktionalistischen Ritualtheorien von Alfred Reginald Radcliffe-Brown und Bronislaw Malinowski.

${ }^{103}$ Didi-Huberman 1996, 77: «Parler d'une fonction des images c'est le plus souvent postuler [...] que les images ont une finalité, qu'elles sont parties intégrantes d'une unité sociale, et qu'enfin [...] que les images seraient tout à la fois des réponses à un besoin social et la représentation même [...] de ce besoin même.» Ib., 79: «epistemologischer Mythos», da ein System von Bedürfnissen vorausgesetzt. Cf. THIEL 1973, 511: Die physiologische Funktion sei auf ein organisches Ganzes ausgerichtet.

${ }^{104}$ Cf. BAUER 1976, 42-44: zur abbildenden Funktion der Architektur. Cf. BANDMANN 1951B und BANDMANN 1969 (Nachdruck von BANDMANN 1951A). 
vermögens für Systemkomplexität das noch verborgene Motiv ist, das im Begriff der Funktion wirksam wird $» .^{105}$

\section{Begriffsgeschichte}

Sollte es in der Geschichtswissenschaft darum gehen, Bedeutungen transkulturell und aus der Vergangenheit in die Gegenwart zu übersetzen, wird es von Vorteil sein, das wissenschaftliche Interesse an eine ähnliche, vergangene Anschauung anzuknüpfen. Je näher die Fachbegriffe an die historischen Bezeichnungen heranrücken, um so weniger verzerrt erscheint das Bild der Geschichte. Die Untersuchung nicht nur der ästhetischen, sondern auch der allgemeinen Bildfunktionen wird dann besonders relevant, wenn der Nutzen, die Wirkung, der Stellenwert oder die Daseinsberechtigung von Kunst in einer bestimmten Epoche debattiert wurden. Dazu gehören beispielsweise die Neudefinition der religiösen Funktionen im Rahmen des byzantinischen Ikonoklasmus, der nordeuropäischen Bilderstürme oder der posttridentinischen Bildreform, wie auch der Festlegung politischer Funktionen im Rahmen totalitärer Ideologien wie Kommunismus und Faschismus. In solchen Zeiten der verstärkten religiösen und politischen Inanspruchnahme von Kunst steht dem Historiker ein enger greifendes Instrumentarium an normativen Funktionsbegriffen zur Verfügung.

Die frühneuzeitliche Geschichte des Begriffs ‘functio> deutet auf ein sich veränderndes Verständnis des Funktionsbegriffs hin. ${ }^{106}$ So wird aus den Schriftquellen deutlich, dass das italienische Wort 〈funzione〉 zunächst nur das öffentliche Amt und die Messliturgie bezeichnete und sich erst im späten 16. und frühen 17. Jahrhundert, im Rahmen der funktionalistischen und rationalistischen Tendenzen in Politik, Religion und Wissenschaft heraus entwickelte. ${ }^{107}$ Denis Diderots und Jean Le Rond d'Alemberts Encyclopédie unterscheidet dann die algebraische Funktion, das heißt die mathematische Relation zwischen veränderlichen Größen, von der aristotelisch verstandenen physiologischen Funktion, nämlich der «Potenz zu Wirken, welche von Organstruktur abhängt, die in Akt verwandelt wird». ${ }^{108}$

\footnotetext{
${ }^{105}$ Niklas Luhmann in SteineR ET AL. 1971-1995. Cf. LuHMANN 1998, 215-301: zur Funktion der Kunst. Cf. Geimer 1998. Cf. De ZurKo 1957в, 238: «Purpose, when thought of as the idea of the artist, the substance of art, permits the extension of functionalism as a concept to all forms of art and offers almost limitless possibilities for application.»

${ }^{106}$ Cf. HiRdina 2000-2001, 591-595: zur Begriffsgeschichte. Cf. GRIMM / GRIMM 1984, vol. 4, 527: Function.

${ }^{107}$ Cf. BAtTAGLiA 1961-1998, vol. 6, 467-468, funzione und StoppelLi / PiCCHI 1997, funzion* und fung*. Cf. THIEL 1973.

108 Diderot / D'Alembert 2000, Fonction: «Fonction (Physiolog.) puissance d'agir qui dépend de la structure de l'organe réduit en acte.»
}

Der künstlerische Funktionsbegriff ist hingegen eine Erscheinung erst des 18. und 19. Jahrhunderts und beschränkt sich meistens auf Architektur- und Designtheorie. ${ }^{109}$ So stellt für Jan Mukarovský «der Funktionalismus die typische Denkweise des Maschinenzeitalters» dar, das mit der industriellen Revolution anbricht und sich im modernen architektonischen Funktionalismus offenbart. ${ }^{110} \mathrm{Ihm}$ folgen Edward Robert De Zurko und Horst W. Janson darin, dass der architektonische Funktionalismus in der Maschinenästhetik der industriellen Revolution und im Rationalismus des 18. Jahrhunderts wurzle. ${ }^{111}$ Erst mit dem Strukturalismus, etwa bei Jan Mukarovský und Jurij Nikolaevic Tynjanov (1894-1943), wird der soziologische und insbesondere von Émile Durkheim geprägte Funktionsbegriff für die Ästhetik und für die Literaturtheorie fruchtbar gemacht. Weil also der neuzeitliche Funktionsbegriff im Cinquecento noch nicht existiert, muss auf verwandte historische Begriffe, wie etwa 〈utilitas〉 und 〈decorum〉, ausgewichen werden, um dem heutigen funktionsgeschichtlichen Blickwinkel auf die Kunst eine ähnliche historische Denkweise gegenüberstellen zu können.

\section{Architektur}

Die Künste werden seit der Antike unter anderem funktional definiert und bewertet. Besonders die Architektur maß und misst man an ihrem Nutzen. 〈Utilitas〉, im Sinne von «Nützlichkeit〉 und 〈Tauglichkeit», spielte seit Vitruvs (um 84- nach 27 v. Chr.) Definition der Architektur als ein Zusammenwirken von firmitas, utilitas und venustas, das heißt von struktureller Festigkeit, praktischer Nutzbarkeit und proportionierter Schönheit, eine fundamentale Rolle in der Planung und Bewertung von Architektur. ${ }^{12}$ Nützlichkeit besteht nach Vitruvs De architectura libri decem in einer einwandfreien Anordnung, die den Gebrauch der Räumlichkeiten nicht behindert, und in einer dem Ort angemessenen und zweckmäßigen Verteilung der Räume. ${ }^{113}$

Vitruvs Begriff 〈utilitas〉 wurde von den italienischen Architekturtheoretikern des 15. und 16. Jahrhunderts übernommen, insbesondere von Leon Battista Alberti (1404-1472) in seiner De re aedificatoria aus der Mitte des

\footnotetext{
${ }^{109}$ Cf. z. B. HiRdina 2000-2001 und SCHNAIDT 1985.

${ }^{110}$ MuKAROVSKÝ 1986, 82: zum Funktionalismus der Architektur als Vorreiter funktionalistischen Denkens. Ib., 83: Der Mensch erlerne den Funktionalismus von der Maschine. Cf. Hirdina 1990.

${ }^{111}$ Cf. De ZurKo 1957в, 46: «The aesthetic of technique did not flourish until the eighteenth century»; es habe in der Renaissance keine Funktionalismusästhetik gegeben. Ib., 233: «The rational view of art is to a large extent responsible for functionalist theory. [...] Rationalism, especially eighteenth-century rationalism [...], opened the door to the principle of functionalism.» Cf. JANSON 1982, 5.

${ }^{112}$ Cf. Grassi / PePe 1978, vol. 2, 623-624: zur utilità. Cf. Jones 1996. Cf. WARNKE 1984, 78-92: zur publica utilitas der mittelalterlichen Architektur.
} 
Quattrocento. ${ }^{114}$ Nach ihm besteht Architektur in einer Verbindung von utilitas, voluptas und dignitas, das heißt Nützlichkeit, Anmut und Angemessenheit. ${ }^{115}$ Er definiert Architektur vor allem auf Grund ihrer Funktionalität, da sie entstanden sei, um existentielle Bedürfnisse der Menschen zu befriedigen. ${ }^{116}$ Folgerichtig ordnet er, je nach necessitas und commoditas, das heißt je nach Bedürfnis und Zweck, Bautypen entsprechenden Menschenklassen zu, weil sie darin unterschiedliche Tätigkeiten ausüben. ${ }^{117}$ Auch Antonio Averlino il Filarete (um 1400-1469) sah in der Mitte des 15. Jahrhunderts den mythischen Ursprung der Architektur in den Grundbedürfnissen des Urmenschen Adam. ${ }^{118}$ Wie David Summers zusammenfasst, spielte die schöne und einheitliche Zweckmäßigkeit der Natur und des menschlichen Leibes die Rolle des organizistischen Paradigmas in der Architekturtheorie der Renaissance, das vom modernen, organizistischen Funktionalismus übernommen wurde, so etwa bei Horatio Greenough (1805-1852). ${ }^{119}$ Nach Caroline van Eck geschieht der Wandel in der Mitte des 19. Jahrhunderts von der aristotelisch geprägten Idee einer «purposive unity», einer «zweckmäßigen Einheit» nach dem Vorbild der gottgeschaffenen Natur, zum nicht teleologischen Begriff der Funktionalität vor allem unter dem Einfluss des darwinistischen Weltbildes. ${ }^{120}$

Der positive Begriff 〈utilis〉 bedeutet, dass ein Raum eine Funktion erfüllt und sich auf Grund seiner Gestalt für einen bestimmten Zweck als nutzbar und nützlich,

${ }^{113}$ VITRUVIUs 1996, 44: «Haec autem ita fieri debent, ut habeatur ratio firmitatis, utilitatis, venustatis [...]; utilitatis autem, [cum fuerit] emendata et sine impeditione usus locorum dispositio et ad regiones sui cuiusque generis apta et commoda distributio.» Ib., 45: «Diese Anlagen müssen aber so gut gebaut werden, dass auf Festigkeit, Zweckmäßigkeit und Anmut Rücksicht genommen wird [...]; auf Zweckmäßigkeit [wird Rücksicht genommen], wenn die Anordnung der Räume fehlerfrei ist und ohne Behinderung für die Benutzung und die Lage eines jeden Raumes nach seiner Art den Himmelsrichtungen angepasst und zweckmäßig ist.» Cf. FonTANA / MorACHIEllo 1975, 83: «E queste cose se debbono fare così, che se habbi rispecto e considerazione alla forteza, all'utilitate, alla venustà e bellezza et alla gratia. [...] Dell'utilitate serrà quan[do] la disposizione serrà emendata senza impedimento a l'uso delli luochi e con atta e comoda distribuzione verso qualunque regione di luoco.» $\mathrm{Cf}$. De ZuRKO 1957в, 26-28: zu Vitruvs organischem Funktionalismus.

${ }^{114}$ Cf. De ZurKo 1957A.

115 AlBERTI 1966, vol. 1, 6: «[l'architettura è un'arte] tale al tempo stesso da conciliare la convenienza pratica [utilitas] con la gradevolezza [voluptas] e il decoro [dignitas]». Cf. De ZurKo 1957в, 47-52: zu Leon Battista Albertis Funktionalismus.

${ }^{116}$ Alberti 1966, vol. 1, 265: «Aedificia hominum esse causa constituta in promptu est.» Ib., 264: «Che gli edifici siano sorti per rispondere ai bisogni degli uomini, è manifesto.»

${ }_{117}$ Alberti 1966, vol. 1, 264-265. Ib., vol. 1, 332-333: zu necessitas und commoditas. Cf. Moos 1974, 210-211 und ROSENBERG 1982.

${ }^{118}$ Filarete 1972, vol. 1, 14-24: zur necessitas des Wohnens als Ursache für die Erfindung der Architektur.

${ }^{119}$ Summers 1981, 319-323. Cf. JOEDICKE 1965, Eck 1994 und GERMAN 1972. Cf. GREenOUGH 1947, S. 73: «I define Beauty as the promise of Function; Action as the presence of Function; Character as the record of Function».

${ }^{120}$ ECK 1994, 268-280: zum «shift to functionality». vorteilhaft und funktional erweist. Darin kommt zum Ausdruck, dass Zwecke die Struktur eines Gebäudes bestimmen sollen und dass in der Architektur von Nutzungskategorien oder Aufgabentypen, also von mehr oder minder normierten, anthropologischen Raumfunktionen ausgegangen wird, wie etwa vom Gottesdienst im Tempel und vom Baden in den Thermen. «Utilitas> konnte im 16. Jahrhundert, wie in Andrea Palladios (1508-1580) I quattro libri dell'architettura von 1570, welche im Entwurfskapitel die dreiteilige Architekturdefinition Vitruvs zitieren, mit 〈comodità〉, das heißt mit ¿Zweckmäßigkeit», 〈Angemessenheit〉 und «Behaglichkeit> gleichgesetzt werden. ${ }^{121}$ Darin scheint sich eine subjektive, sinnliche Perspektive abzuzeichnen: «Comodità $>$ macht den wahrnehmbaren Vorzug der Funktionalität, die Annehmlichkeit selbst, zu einer Aufgabe der Architektur, wie sie im heutigen Komfortbegriff weiterbesteht. Auch in der Praxis ist in der frühen Neuzeit eine Zunahme an funktionalen Interessen deutlich zu beobachten, etwa im Entwurf von Idealstädten, in der Entwicklung der Militärarchitektur, in der Spezialisierung von höfischen Raumfunktionen. ${ }^{122}$ Man wird also in der Architekturtheorie und -praxis des Cinquecento von einem verbreiteten Bewusstsein für Funktionalismus sprechen dürfen. Der funktionsgeschichtliche Ansatz ist daher in der Architekturforschung seit langem etabliert.

\section{Malerei}

Die Malerei wurde ebenfalls unter anderem funktional definiert. So zählte Leon Battista Alberti ihre Vorzüge und Zwecke auf. ${ }^{123}$ Zwar erhielt besonders die Architektur, auf Grund des Bauens als menschliches, gesellschaftliches und politisches Primärbedürfnis, das Epitheton 〈utilis〉, doch sollten im 16. Jahrhundert die bildenden Schwesternkünste diesem durch die Antike beglaubigten Qualitätssiegel nacheifern und ebenfalls an ihrem Nutzen gemessen werden wollen. Beispielsweise versuchte der Kunsttheoretiker Benedetto Varchi (1503-1565) in seinen Vorlesungen Della maggioranza delle arti von 1547 die sowohl nützlichen wie auch angenehmen arti del disegno funktional zu hierarchisieren:

${ }^{121}$ Palladio 1997, 6: «Tre cose in ciascuna fabrica (come dice Vitruvio) deono considerarsi, senza le quali niuno edificio meriterà esser lodato; \& queste sono, l'utile, ò commodità, la perpetuità, \& la bellezza $[. .$.$] . La commodità si havrà, quando à ciascun membro$ sarà dato luogo atti, sito accomodato, non minore che la dignità si ricchiegga, ne maggiore che l'uso si ricerchi: \& sarà posto in luogo proprio, cioè quando le loggie, le sale, le stanze, le cantine, e i granari saranno posti a' luoghi loro convenevoli.» Cf. GRASSI / PEPE 1978, vol. 1, 119-120: zur comodità. Cf. n. 105: zur commoda distributio. Cf. DE ZuRKо 1957в, 54-55: zu Palladios Funktionalismus.

122 PARAvicini 1997, 13: "Allenthalben ist Funktionsdifferenzierung zu beobachten.» Cf. ib., 22-25.

${ }_{123}$ Alberti 1975, 44-46. 
"In den Künsten muss man vor allem ihren Zweck beachten. So wie der Zweck mehr oder minder würdig ist, wird auch die jeweilige Kunst mehr oder minder nobel sein.» ${ }^{124}$ Dabei spricht er der Architektur, wie die Tradition es will, den höchsten Rang zu, nämlich «auf Grund ihrer großen Nützlichkeit», ihrer utilità. ${ }^{125}$ Darin zeigt sich, dass der Funktionsbegriff durchaus ein Werturteil implizieren kann. Auch die platonische Frage, ob Zweckmäßigkeit eine hinreichende Bedingung für Schönheit sei, wurde, laut David Summers, im Cinquecento zur Debatte gestellt, etwa von Vincenzo Danti (1503-1565), der die Naturschönheit als wahrgenommene Zweckmäßigkeit verstand: «dal fine dipende la bellezza». ${ }^{26}$

Die Malerei wurde im Zuge der katholischen Bildreform einer funktionalistischen Neubestimmung im Dienste des Glaubens unterworfen, welche die Rechte und Pflichten der Kunst auf Grund ihrer Nutzbarkeit für die Konfessionalisierung neu definierte. ${ }^{127}$ So spricht etwa der richtungweisende Bologneser Bildreformer Gabriele Paleotti (1522-1597) in seinem Discorso intorno alle immagini sacre e profane aus dem Jahre 1582 wiederholt vom Nutzen der Malerei. Ein christliches Bild bestehe aus drei Teilen, dem Angenehmen, dem Nützlichen und dem Guten, «il dilettevole, l'utile e l'onesto». ${ }^{128}$ Dem Gemälde kommen nach Gabriele Paleotti vielerlei Zwecke zu, und Bilder bestünden zuerst darin, dem Dargestellten zu ähneln. Die Hauptaufgabe des Malers sei jedoch, mit Kunst und Mühe die Gnade Gottes zu erlangen. ${ }^{129}$ Daher habe auch die pittura cristiana, die christliche Malerei, den eigentlichen und höheren Zweck, über die bloß unterhaltende Naturnachahmung hinaus, die unvergängliche Glorie Gottes anzublicken und anzustreben, indem sie durch ihre Bilder die Menschen vom Laster abbringe und zur wahren Gottesverehrung hinwende. ${ }^{130}$

${ }^{124}$ VARCHI 1960-1962, 11: «Diciamo dunque che nelle arti si debbe attendere principalmente e considerare il fine, e secondo che il fine è o meno o più degno, così l'arte è più o meno nobile.» $C f$. ib., $5-6$.

${ }^{125}$ VARCHI 1960-1962, 21: «per la sua grande utilità».

${ }^{126}$ DANTI 1962, 224: «che la bellezza propriamente si vede e risplende nelle membra et altre cose atte a conseguire il loro fine».

${ }^{127}$ Cf. Grassi / Pepe 1978, vol. 2, 623. Cf. z. B. Weddigen 2000.

${ }^{128}$ Paleotti 1961, 213. Cf. ib., 138: «onesto, utile e dilettevole».

${ }^{129}$ PALEOTti 1961, 210: «Il fine della pittura serà l'assomigliare la cosa rappresentata.» Ib.: «Il fine principale [del pittore] serà, col mezzo della fatica et arte sua acquistarsi la grazia divina.»

${ }^{130}$ Paleotti 1961, 211: «Si che, quanto al proposito nostro, la pittura, che prima aveva per fine solo di assomigliare, ora, come atto di virtù, piglia nuova sopraveste, et oltre l'assomigliare si inalza ad un fine maggiore, mirando la eterna gloria e procurando di richiamare gli uomini dal vizio et indurli al vero culto di Dio.»

\section{Dekorum}

Die Funktionalisierung der Künste durch die katholische Reform wertete einen weiteren Fachterminus auf: Mit der Funktion und dem Nutzen ist das Dekorum verwandt. Der aristotelische, rhetorische Begriff «decorum > gehört sowohl in der Antike wie auch in der Renaissance zu den wichtigsten ethischen und ästhetischen Urteilskategorien. Beispielsweise bezeichnete Vitruv mit 〈decor〉 die Zweckmäßigkeit der Architektur, und für Leonardo da Vinci (1452-1519) soll die Darstellung der Menschen auf ihrem Geschlecht, Alter, Stand und Charakter angemessen sein. ${ }^{131}\langle$ Decoro $\rangle$ - im Sinne von «Angemessenheit - meint, wie «Nutzen` und «Funktion>, eine bloße Relation, die alle Lebensbereiche betreffen kann: Eine Sache ist einer anderen angemessen, und sie stehen in einem Proportionsverhältnis zu einander, das für ein Drittes, für einen höheren Zweck, sich als günstig erweist.

Daher konnten Traktatschreiber der katholischen Reform mit «decoro〉 sowohl ästhetische wie auch moralische Aspekte der Kunst unter einen Begriff fassen. ${ }^{132}$ Dass dem Dekorum ein Proportionalitätsgedanke zu Grunde liegt, belegt etwa Gabriele Paleottis Benennung der Bilder, die einen für ihre religiöse Funktion unangemessenen und daher unmoralischen Anteil unterhaltsamer Kunstfertigkeit zur Schau stellen, als «pitture sproporzionate», als unproportionierte Gemälde. ${ }^{133} \mathrm{Ga}-$ briele Paleotti ging in seinem Abschnitt über die unschicklichen und unangebrachten Bilder, Delle pitture inette ed indecore, so weit, die allgemeine und verbreitete Bedeutung des decorum, nämlich als verisimilitudo und wirklichkeitsnaher Angemessenheit einer Darstellung, ganz von der moralischen, für ihn primären Bedeutung des decorum abzutrennen, das die Würde einer Person, ihre dignitas bezeichnet. ${ }^{134}$ Als verwandte Funktionali-

${ }^{131}$ Fontana / Morachiello 1975, 81: zu Vitruv. Cf. Aristoteles 1982, Abs. 15: zur Angemessenheit des dargestellten Charakters. Cf. ib., Abs. 17: zum Passenden. Cf. VINCI 1882, 188, no. 371 / 377: zur «Wohlanständigkeit». Cf. RUtHERFord / Mildner 1996-2001: zum Dekorum. Ib., 434: «In beiden Bereichen [Malerei und Architektur] ist das Dekorum wegen seiner einheitsstiftenden Wirkung eine Lehre von der Erscheinungsweise des Individualschönen, entsprechend den Inhalts- und Funktionsmerkmalen der darzustellenden Sache. Als das dem Augenblick (kairos) Angemessene, Geziemende, Charakteristische bezieht sich das Dekorum sowohl auf die Binnenstruktur eines Werkes wie auf seine Außenwirkung. Als das Ordnende, Zuordnende und Geordnete gliedert es die Teile zu einem zweckmäßigen, sinnvollen und harmonischen Ganzen, wobei die spezielle Wirkungsweise des einheitlichen Eindrucks im subjektiv Stimmigen wie objektiv Nachvollziehbaren liegt.» Cf. GRASSI / PEPE 1978, vol. 1, 144-145: zum decoro. Cf. GAStON 1996. De ZurKo 1957в, 29-30: bei Quintilian sei Schönheit nie von Nützlichkeit getrennt. Cf. BIERMANN 1997.

${ }^{132}$ Cf. Blunt 1962, 35 und 122

${ }^{133}$ Paleotti 1961, 374-379, cap. Delle pitture sproporzionate.

${ }^{134}$ PALEOTti 1961, 372: «Ma noi [...] ci appigliamo al parere de' più savi, che attribuiscono il decoro propriamente alla dignità della persona.» 
tätsbegriffe in der italienischen Kunsttheorie der frühen Neuzeit sind des Weiteren $<$ fine >, <proporzione〉, ‘convenienza oder $\left\langle\right.$ dignità $>$ zu nennen. ${ }^{135}$

Der Funktionsgedanke lässt sich freilich, über die Kunsttheorie hinaus, ebenfalls in der Kunstpraxis des 16. Jahrhunderts belegen. Zum Beispiel sind in unzähligen Architekturzeichnungen funktionale Raumbegriffe, wie etwa <anticamera oder $\langle$ cubicolo〉, eingetragen, nach welchen der Grundriss organisiert wird. ${ }^{136}$ Diese deuten auf konventionelle Raumnutzungen und soziale Verhaltensformen hin, welche jedoch flexibel blieben: Räume, wie etwa der salone, wurden multifunktional benutzt und dementsprechend ad hoc möbliert. ${ }^{137}$ Auch die wenigen erhaltenen, schriftlichen Dekorationsprogramme beziehen sich gelegentlich explizit auf Raumnutzungen. Es sei hier nur auf das Beispiel von Annibale Caros (1507-1566) Programm von 1562 hingewiesen, welches Taddeo Zuccaro (1529-1566) im Farnesepalast zu Caprarola in einem Deckengemälde umsetzen sollte: Das Schlafzimmer des Hausherren, des Kardinals Alessandro Farnese (1545-1592), müsse mit einem der Raumfunktion angemessenen Sujet, "cose convenevoli al luogo», dekoriert werden, also mit Allegorien des Schlafes. ${ }^{138}$

Obwohl 〈funzione〉 erst spät auftaucht, findet der Funktionalismus in den frühneuzeitlichen Begriffen wie 〈utilità und 〈decoro eine Entsprechung. Nicht nur war diese Möglichkeit, einen zweckmäßigen Bezug zwischen dem Menschen und seiner ästhetischen Um-

${ }^{135}$ Cf. Alberti 1975, 78: zum conveniente. Cf. ib., 19: zum condecens. Cf. Grassi / PePe 1978, vol. 1, 128-129: zur convenienza. Cf. ib., vol. 2, 434-438: zur proporzione. Cf. AlberTI 1975, 66: «Poi appresso ogni cosa seguiti dignità.» Cf. ib., 67: «Tum et pro dignitate omnia subsequentur.» Cf. GRASSI / PePE 1978, vol. 1, 150: zur dignità. Cf. HAusHERR 1984: zur historisch korrekten Darstellung.

${ }^{136}$ Cf. Frommel 1973, vol. 3: Beispiele für das 15. und 16. Jahrhundert. Cf. WADDY 1990: Beispiele für das 17. Jahrhundert.

${ }^{137}$ WARNCKe 1997. Cf. HeRKLOtz 1990, 225: zur Aufbarung eines verstorbenen Kardinals im salone.

${ }^{138}$ Annibale Caro in VASARI 1906, vol. 7, 115: «Quanto alla camera della volta piatta [...] mi pare, che, essendo ella destinata per il letto della propria persona di Sua Signoria illustrissima, vi si debbano fare cose convenevoli al luogo.» gebung herzustellen, wie etwa Paolo Cortesis (14711510) De cardinalatu von 1510 für den Palastbau bestätigt, grundlegend für Kunstproduktion und -rezeption, sondern bestimmte, nach dem Vorbild der antiken Rhetorik, jeden Lebensbereich, wie etwa Baldassare Castigliones (1478-1529) epideiktische Streitgespräche zeigen, in denen er die unterschiedlichsten Dinge, seien es Geschlechter, Sportarten oder Künste, nach ihrer relativen utilità abwägt. ${ }^{139}$

Die Relativität, die dem Funktionsbegriff inhärent ist, war einerseits im christlichen Gedankengut vorgeprägt, das die Dinge nicht für sich bewertet, sondern mit einem Übergeordneten oder Absoluten in Bezug setzt, und andererseits in den Ideen und Praktiken von Wissenschaft und Kunst, die seit dem Mittelalter vor allem auf der geometrischen und mathematischen Proportionalität und deren Berechnung und Schätzung beruhten. ${ }^{140}$ Die Rationalisierung staatlicher und kirchlicher Organe, die Festschreibung von Zeremoniell und Liturgie, die Entdeckung fremder Kulturen und die Wiedergeburt der Antike, die Ausbildung des Hoflebens, der Gebrauch der genannten Termini wie «utilità und $<d e-$ coro - nur um einige wenige Phänomene zu nennen deuten darauf hin, dass in der frühen Neuzeit mit einem verankerten und sich entwickelnden Begriff der Relativität und Funktionalität zu rechnen ist, der eine funktionsgeschichtliche und kontextuelle Analyse von frühneuzeitlichen Kunstwerken und Gegenständen nicht bloß erlaubt, sondern geradezu erfordert.

\footnotetext{
${ }^{139}$ Cf. Cortesi 1980 und Weil-Garris / D'Amico 1980, 54: zu den verschiedenen Funktionstypen bei Leon Battista Alberti. Cf. CASTIGLIONE 1960, 344: «se considerate tutte le cose, trovarete che sempre quelle che son bone ed utili hanno ancor grazia di bellezza». Cf. ib., 345: «Sostengon le colonne e gli architravi le alte logge e palazzi, né però son meno piacevoli agli occhi di chi le mira, che utili agli edifici.»

${ }^{140}$ Cf. BaXandall 1984, 112-137. Cf. Vogt 1977, 30: zum Ursprung des Funktionalismus im Newtonianismus und der naturwissenschaftlichen Aufklärung, mit Wurzeln in der Reformation und Renaissance. Cf. Ullmann 1960: zum Funktionalismus im Papsttum und Kaisertum.
} 